\title{
A POLARIMETRIC SURVEY FOR DUST IN 47 TUCANAE $^{1}$
}

\author{
Juan C. Forte, ${ }^{2}$ Lilia P. Bassino, ${ }^{2,3}$ E. Irene Vega, ${ }^{2,4}$ Leonardo J. Pellizza González, ${ }^{4}$ \\ Sergio A. Cellone, ${ }^{2.3}$ and Mariano R. Méndez ${ }^{5}$ \\ Received 2002 February 6; accepted 2002 February 28
}

\begin{abstract}
We present linear polarization measurements in the $V$ band for 77 stars in the field of the globular cluster 47 Tucanae (NGC 104) and for 14 regions free of bright stars, located along an elliptical isophotal contour of the cluster, as well as $U B V R I$ measurements for the cluster nucleus. The observations show variable foreground polarization that, once removed, leaves marginally significant polarization residuals for the nonvariable bright red giants. Although these residuals are small, there is a systematic trend in the sense that the larger ones are seen toward the south of the cluster (in a direction opposite to that of the cluster proper motion). In contrast, most of the variable stars do show significant intrinsic polarization. The behavior of the star-free regions is similar to that of the nonvariable stars and sets an upper limit to the possible existence of a global pattern of scattered (and polarized) intracluster light in the $V$ band. In turn, the multicolor observations of the cluster nucleus cannot be fitted with a Serkowski law and exhibit a polarization excess in both $U$ and $B$. This polarization could be explained as a combination of the foreground interstellar component and another component arising from dust located in the nucleus and illuminated by a bright blue post-asymptotic giant branch star (at $48^{\prime \prime}$ from the cluster center). Inspection of a set of archival Hubble Space Telescope Wide Field Planetary Camera 2 images reveals the presence of a number of dark patches in the innermost regions of the cluster. A prominent patch (some $5^{\prime \prime} \times 3^{\prime \prime}$ in size) located $12^{\prime \prime}$ from the cluster center and with a position angle (north to east) of $120^{\circ}$ has a slightly different polarization compared with that of the cluster nucleus and appears to be a good candidate for identification as a dust globule within the cluster.
\end{abstract}

Key words: dust, extinction — globular clusters: individual (NGC $104=47$ Tucanae) — polarization

\section{INTRODUCTION}

Arguments in favor of and against the presence of dust within globular clusters have been given along the years. Although an increasing volume of recent infrared observations suggest that the dust content should be very small, if any, the number of hypotheses regarding the nature of the dust particles (presumably originating from mass-loss processes in luminous red giants), their spatial distribution, equilibrium temperatures, etc., render these dust mass estimates still uncertain. A good example of this situation is depicted in the classic paper by Gillett et al. (1988), who discuss IRAS observations of the globular cluster 47 Tucanae (NGC 104) and report the existence of excess infrared emission at $100 \mu \mathrm{m}$, attributed to thermal dust radiation. That result can be confronted with more recent Infrared Space Observatory (ISO) observations that reach $120 \mu \mathrm{m}$ (Hopwood et al. 1999) and do not confirm the presence of such excess. Both papers are illustrative of the difficulties, among others, involved in the subtraction of the stellar background (see also Knapp, Gunn, \& Connolly 1995).

Among the arguments in favor of dust within globular clusters, starting with the statistical approach of Roberts

\footnotetext{
${ }^{1}$ Based on observations made at the Complejo Astronómico El Leoncito, which is operated under agreement between CONICET and the National Universities of La Plata. Córdoba, and San Juan.

2 Facultad de Ciencias Astronómicas y Geofísicas. Universidad Nacional de La Plata, Paseo del Bosque s/n, 1900 La Plata, Argentina; and CONICET; forte@fcaglp.unlp.edu.ar.

${ }^{3}$ Instituto de Astrofísica de La Plata. CONICET.

4 Instituto de Astronomía y Física del Espacio. CONICET. C.C. 67. Sucursal 28, 1428 Buenos Aires, Argentina.

5 Space Research Organization Netherlands, Sorbonnelaan 2, NL-3584 CA Utrecht, Netherlands.
}

(1960), we can mention the multicolor photometric observations by Kanagy \& Wyatt (1978) and Forte \& Méndez (1988), or the polarimetry by Martin \& Shawl (1981) and Forte \& Méndez (1989). In turn, Aurière \& Leroy (1990) disputed these results and pointed out that, in some cases, neither the photometric nor the polarimetric observations could yield an unambiguous answer to the nature of the dark patches seen in several globular clusters, that is, whether they are real dust clouds or just statistical fluctuations of the stellar distribution or, alternatively, if the dust is inside or simply along the line of sight to the cluster.

This paper presents a linear polarization survey of the globular cluster 47 Tuc. Because of its high metallicity and high mass, this cluster appears to be a good candidate to form and retain (at least some) dust. The structure of the survey includes three different components:

1. The search for intrinsic polarization in the most luminous red giants, which, as a result of mass-loss processes, could form and eject dust particles. The integrated scattered light will be polarized only if the resulting dust shells from those processes are not spherically symmetric around the star. This kind of survey has been carried out (with negative results) by Minniti, Coyne, \& Tapia (1990) and Minniti, Coyne, \& Clariá (1992) and, in the case of 47 Tuc itself, by Origlia et al. (1997), who however mention the possible detection of intrinsic polarization in some of its red variable stars.

2. The search for tangential polarization (i.e., perpendicular to the direction to the cluster center at a given position) arising from dust illuminated by the overall radiation field. The use of this effect as a diagnostic tool for dust within stellar systems was originally proposed by Jura (1978). For example, the existence of low optical depth dust distributed 
around the cluster center (and following some kind of radial dependence) would produce a regular pattern in the polarization $P$, that is, a double-wave behavior of the $U$ and $Q$ Stokes parameters, defined as $U=P \sin 2 \theta$ and $Q=P \cos 2 \theta$, with position angle on the sky. For this purpose, a number of regions free of bright stars were observed that follow, approximately, an isophotal contour of the cluster (no star brighter than $V \sim 15.0$ could be seen within the diaphragm).

3. A multicolor analysis of the cluster nucleus using several different-aperture diaphragms, ranging from $17^{\prime \prime}$ to $45^{\prime \prime}$ in diameter. Any wavelength dependence of the polarization due to interstellar dust along the line of sight to the cluster should be typified by a Serkowski (1973) law. Alternatively, a deviation from that law might indicate the presence of excess polarization with a different wavelength dependence, as would be expected, for example, in the case of light scattered by dust particles.

\section{OBSERVATIONS}

Linear polarization observations were carried out in September or October during 1997, 1998, 1999, 2000, and 2001 runs with the $2.15 \mathrm{~m}$ telescope at the Complejo Astronomico El Leoncito (San Juan, Argentina) and a rotatingplate polarimeter. This instrument is an improvement on the original designs known as MINIPOL and VATPOL (see Magalhães, Benedetti, \& Roland 1984) and takes advantage of two high-throughput photocells (Martínez et al. 1990). Standard stars for polarization were observed in all runs, with the aim of determining both the instrumental polarization (less than $0.02 \%$ in all the UBVRI filters) and the zero point of the polarization angle system.

Visual polarizations were measured using a $17^{\prime \prime}$ diaphragm centered at the positions of bright red giants; that is, the output values correspond not only to the stars but also to the fainter stellar background within the diaphragm. No attempt was made to correct for the local background, since it varies in a strong way with position within the cluster, and instead, a blank region located $45^{\prime}$ to the south of the cluster center was repeatedly observed during each night in order to determine the temporal variation of the sky polarization. Integration times ranged from 10 to 15 minutes per observation. The rms error of the polarization, estimated according to Magalhães et al. (1984), as a function of magnitude, can be well represented as $\sigma_{P_{V}}=(0.015 \mathrm{~V}-0.117) \%$, where $V$ is the magnitude corresponding to the total brightness within the $17^{\prime \prime}$ diaphragm. For the whole sample, we obtain a median rms error $\sigma_{P_{V}}= \pm 0.045 \%$, which leads to a typical uncertainty of about $\pm 3^{\circ}$ in the polarization angles. Corrections to the observed polarizations due to noise bias were estimated following Clarke \& Stewart (1986) and were neglected, since, in the worst cases, they were smaller than $0.01 \%$.

The bright-star-free regions are located approximately along the isophotal contour of the cluster corresponding to a major semiaxis of $110^{\prime \prime}$, that is, some 4.6 core radii, adopting a core radius of $24^{\prime \prime}$ (see, e.g., Howell, Guhathakurta, \& Gilliland 2000). These regions were also observed with a $17^{\prime \prime}$ diaphragm and integration times of 15 minutes each, yielding a typical polarization error of $\sigma_{P_{Y}}= \pm 0.05 \%$.

Polarimetry of the cluster nucleus was obtained through $U B V$ (Johnson) and $R I$ (Kron-Cousins) filters during the
1997 run. In this case, the observations were carried out through diaphragms with diameters of $17^{\prime \prime}, 33^{\prime \prime}$, and $45^{\prime \prime}$ and integration times from 5 to 15 minutes for each observation. In total, we obtained eight integrations in the $V$ and $R$ bands and five for each of the remaining filters. Neutral filters were added for the $V R I$ observations in order to avoid saturation due to the brightness of the cluster nucleus. Sky polarization measurements for the bright-star-free regions and for the nucleus were also secured on the blank region $45^{\prime}$ to the south of the cluster.

Approximate $V$ magnitudes were determined for the total brightness observed through the $17^{\prime \prime}$ diaphragm according to the formula $V=v_{i}+C_{V}$, where $v_{i}$ is the instrumental visual magnitude (corrected for atmospheric extinction) and $C_{V}=0.19 \pm 0.05$ is a constant derived by means of 19 stars included in the photometry by Lee (1977). These stars are far from the cluster center and not affected by crowding. No color term was added, on the basis that most of the program stars have color indexes within the color range covered by the calibrating stars.

\section{OBSERVED POLARIZATIONS}

\subsection{Nonvariable Stars}

Figure 1 shows a finding chart for 53 stars (not reported as variables) located within $4^{\prime}$ from the cluster center, whose observed $V$ polarizations are presented in Table 1 and shown on the sky in Figure $2 a$. The table gives the star identification number, coordinates (J2000), the approximate $V$ magnitude measured within the $17^{\prime \prime}$ diaphragm, the observed linear visual polarization, associated error, and polarization angle (north to east). These data are followed by the star identification and membership probability MP (when available) from the proper-motion study by Tucholke (1992), as well as the identification, $V$ magnitude, and $B-V$ color from Chun \& Freeman (1978) and, in the last column, the heliocentric radial velocity from Mayor et al. (1983).

From the 53 stars listed in Table 1, 44 seem to be bright red giants and cluster members, according to their position in the $V$ versus $B-V$ diagram. This number increases to 46 if we add one star with a high membership probability from its proper motion (star 43, with no photometry available) and include an early-type post-asymptotic giant branch (AGB) star labeled BS by Lloyd Evans (1974) and UIT 7 by O'Connell et al. (1997); see also Dixon, Davidsen, \& Ferguson (1995). For this particular star, we obtain $P_{V}=0.37 \% \pm 0.02 \%$ and $\theta_{V}=123 \%$. In turn, 39 of the observed stars have radial velocities that are consistent with membership and follow the cluster rotation (when plotted against their linear coordinates along the major axis of the cluster; see Mayor et al. 1984).

We note that even though six stars have low membership probability from their proper motions (MP index lower than 0.2 ), neither their position in the color-magnitude diagram nor their polarization values exhibit significant differences from other stars considered to be cluster members and, for this reason, were kept within the sample.

Table 2 extends the polarization observations to 14 other stars located at angular distances between $4^{\prime}$ and $20^{\prime}$ from the cluster center. This table gives only approximate rectangular positions with respect to the cluster center ( $X$ and $Y$ positive toward the east and north, respectively). All these stars have two-color photometry from Lee (1977) indicating 


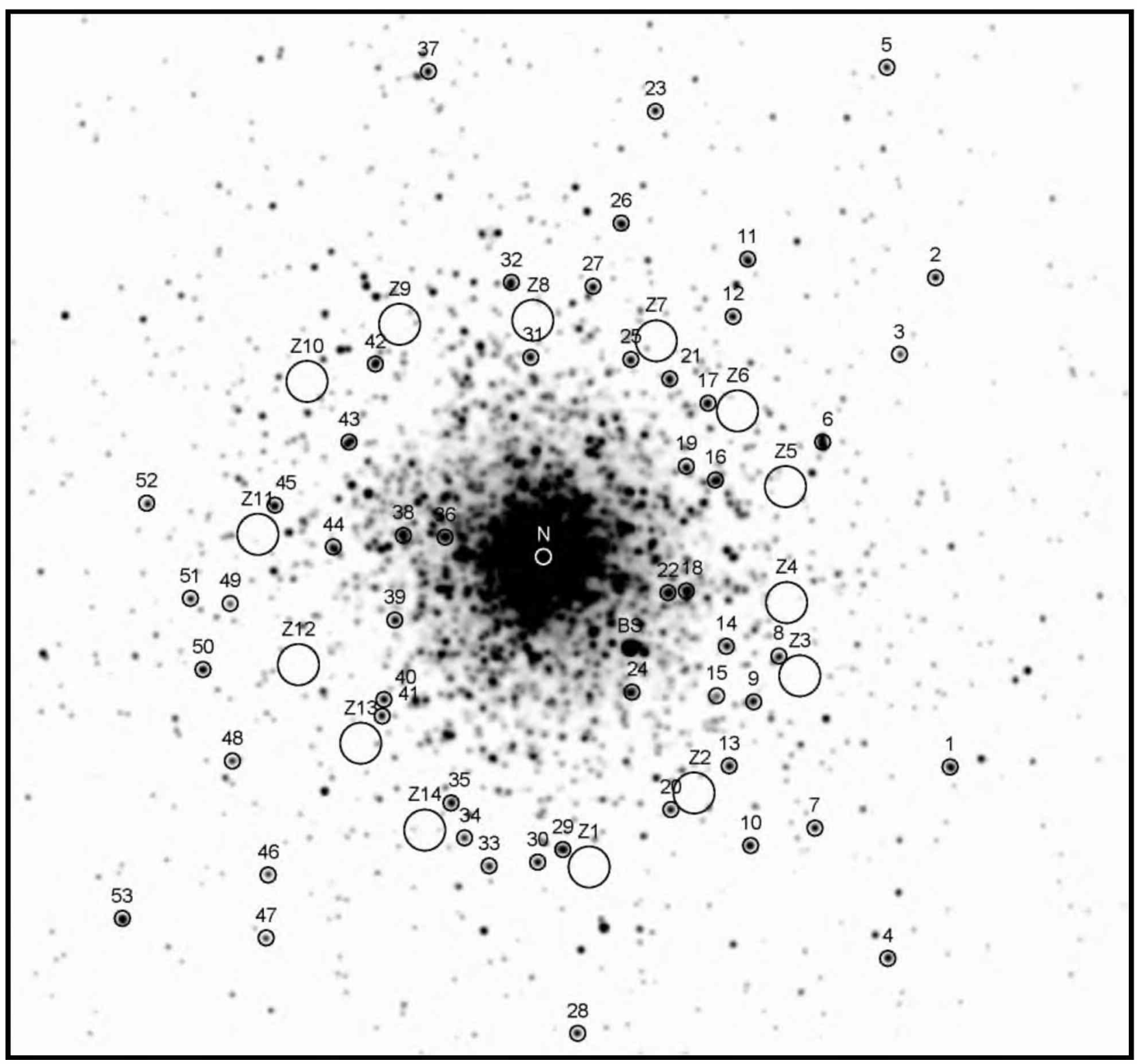

FIG. 1.-Identification chart for the observed giant stars in 47 Tuc. North is up, and east is to the left. The bright early post-AGB star is labeled BS (see text). Larger circles correspond to the bright-star-free regions.

that they are probable cluster members. One of them, L1427, as discussed below, seems to be associated with a long tail-like structure detected on the IRAS raster image presented by Gillett et al. (1988).

\subsection{Variable Stars}

The observed polarizations for the variable stars are given in Table 3. The identification numbers come from Sawyer Hogg (1973). One of these objects (V4) shows discrepant values when comparing the 1997 and 1999 data; both sets are given in Table 3, as we cannot reject a possible variation of the polarization.

\subsection{Bright-Star-free Regions}

The polarizations corresponding to the bright-star-free regions are listed in Table 4 and displayed on the sky in
Figure $2 b$. That table also includes the $\mathrm{J} 2000$ coordinates, approximate integrated $V$ magnitudes, and position angles on the sky measured at the cluster center (north to east).

\subsection{Cluster Nucleus}

The $U B V R I$ observations of the cluster nucleus are listed in Table 5. The adopted coordinates for the cluster center are $\alpha=00^{\mathrm{h}} 24^{\mathrm{m}} 5.95$ and $\delta=-72^{\circ} 04^{\prime} 53^{\prime \prime}(\mathrm{J} 2000)$, within $2^{\prime \prime}$ of the center coordinates estimated by Guhathakurta et al. (1992). Even though the polarizations were obtained using three different diaphragms, the $B V R I$ values do not show a significant trend with aperture, and thus we only give the average values for each of these bands. The ultraviolet measures do show significant differences in polarization with aperture (see $\S 7$ ), and in this case the individual values for each diaphragm are given. 
TABLE 1

Visual Polarizations for Bright Giant Stars in 47 Tucanae

\begin{tabular}{|c|c|c|c|c|c|c|c|c|c|c|c|}
\hline No. & $\begin{array}{c}\text { R.A. } \\
(\mathrm{J} 2000)\end{array}$ & $\begin{array}{c}\text { Decl. } \\
(\mathrm{J} 2000)\end{array}$ & $\begin{array}{c}V_{17^{\prime \prime}} \\
\text { (mag) }\end{array}$ & $\begin{array}{l}P_{V} \\
(\%)\end{array}$ & $\begin{array}{c}\theta_{L^{r}} \\
(\operatorname{deg})\end{array}$ & Tucholke & MP & $\begin{array}{l}\text { Chun \& } \\
\text { Freeman }\end{array}$ & $\begin{array}{c}V^{r} \\
\text { (mag) }\end{array}$ & $\begin{array}{c}B-V \\
\text { (mag) }\end{array}$ & $\begin{array}{c}\text { R.V.V. } \\
\left(\mathrm{km} \mathrm{s}^{-1}\right)\end{array}$ \\
\hline $1 \ldots \ldots$. & 002329.4 & -720620 & 11.27 & $0.47 \pm 0.05$ & 128.6 & 1175 & 98.3 & $\mathrm{E} 175$ & 11.63 & 1.86 & -4.4 \\
\hline $2 \ldots \ldots$ & 002330.9 & -720257 & 11.64 & $0.37 \pm 0.06$ & 120.8 & 1191 & 96.9 & $\mathrm{E} 275$ & 12.06 & 1.48 & -27.9 \\
\hline $3 \ldots \ldots$. & 002334.1 & -720329 & 12.02 & $0.31 \pm 0.06$ & 125.5 & $1225^{a}$ & 96.5 & $\mathrm{E} 270$ & 12.87 & 1.27 & -35.8 \\
\hline $4 \ldots \ldots$ & 002335.0 & -720739 & 11.43 & $0.30 \pm 0.05$ & 125.2 & $\ldots$ & $\ldots$ & E97 & 11.73 & 1.74 & -10.1 \\
\hline $5 \ldots \ldots$ & 002335.3 & -720130 & 11.67 & $0.41 \pm 0.06$ & 121.1 & 1243 & 23.7 & E323 & 12.12 & 1.51 & -11.5 \\
\hline $6 \ldots \ldots$ & 002340.9 & -720405 & 10.62 & $0.43 \pm 0.04$ & 125.2 & $\ldots$ & $\ldots$ & $\ldots$ & $\ldots$ & $\ldots$ & $\ldots$ \\
\hline $7 \ldots \ldots$ & 002341.6 & -720645 & 11.16 & $0.35 \pm 0.05$ & 122.8 & 1320 & 0 & E90 & 12.32 & 1.40 & -10.3 \\
\hline $8 \ldots \ldots$ & 002344.8 & -720534 & 11.61 & $0.41 \pm 0.06$ & 132.6 & $\ldots$ & $\ldots$ & F111 & 12.22 & 1.37 & +1.6 \\
\hline $9 \ldots \ldots$. & 002347.1 & -720553 & 10.99 & $0.34 \pm 0.05$ & 121.3 & $\ldots$ & $\ldots$ & F86 & 12.03 & 1.49 & -6.0 \\
\hline $10 \ldots \ldots$ & 002347.3 & -720653 & 11.31 & $0.31 \pm 0.05$ & 130.0 & $\ldots$ & $\ldots$ & E83 & 12.08 & 1.58 & -22.3 \\
\hline $11 \ldots$. & 002347.7 & -720249 & 11.15 & $0.36 \pm 0.05$ & 123.1 & 1379 & 0 & E304 & 11.69 & 1.82 & -27.8 \\
\hline $12 \ldots$. & 002349.0 & -720313 & 11.42 & $0.30 \pm 0.06$ & 123.5 & $\ldots$ & $\cdots$ & F218 & 12.13 & 1.49 & -13.0 \\
\hline $13 \ldots$. & 002349.3 & -720620 & 11.24 & $0.41 \pm 0.05$ & 130.4 & 1390 & 98.3 & F66 & 12.00 & 1.50 & -8.3 \\
\hline $14 \ldots \ldots$ & 002349.5 & -720530 & 11.05 & $0.38 \pm 0.05$ & 120.0 & $\ldots$ & $\ldots$ & F106 & 12.07 & 1.50 & -5.6 \\
\hline $15 \ldots \ldots$ & 002350.4 & -720551 & 11.16 & $0.38 \pm 0.06$ & 130.0 & $\ldots$ & $\ldots$ & F89 & 12.21 & 1.69 & -13.5 \\
\hline $16 \ldots$. & 002350.5 & -720421 & 10.45 & $0.38 \pm 0.04$ & 124.6 & ... & $\cdots$ & F188 & 11.48 & 2.28 & -26.8 \\
\hline $17 \ldots$. & 002351.2 & -720349 & 10.82 & $0.44 \pm 0.04$ & 121.4 & $\cdots$ & $\ldots$ & F203 & 11.70 & 1.77 & -7.7 \\
\hline $18 \ldots$. & 002353.1 & -720507 & 10.11 & $0.34 \pm 0.03$ & 121.0 & $\therefore$ & $\ldots$ & $\ldots$ & ... & $\ldots$ & $\ldots$ \\
\hline $19 \ldots \ldots$ & 002353.1 & -720415 & 10.56 & $0.35 \pm 0.04$ & 120.6 & $\ldots$ & $\ldots$ & F193 & 11.62 & 1.86 & -29.1 \\
\hline $20 \ldots$. & 002354.5 & -720638 & 11.29 & $0.39 \pm 0.05$ & 116.4 & $\ldots$ & $\ldots$ & F60 & 12.34 & 1.37 & -14.0 \\
\hline $21 \ldots$. & 002354.6 & -720339 & 10.94 & $0.48 \pm 0.05$ & 123.3 & ... & $\ldots$ & F229 & 11.71 & 1.76 & -31.4 \\
\hline $22 \ldots$. & 002354.8 & -720508 & 10.97 & $0.36 \pm 0.05$ & 123.8 & $\ldots$ & ate & $\ldots$ & t.. & $\ldots$ & $\ldots$ \\
\hline $23 \ldots \ldots$ & 002356.0 & -720148 & 11.57 & $0.43 \pm 0.06$ & 121.1 & ... & $\ldots$ & E354 & 12.44 & 1.35 & -14.9 \\
\hline $24 \ldots \ldots$ & 002358.0 & -720549 & 10.64 & $0.39 \pm 0.05$ & 122.0 & $\ldots$ & $\ldots$ & $\ldots$ & ... & $\ldots$ & $\ldots$ \\
\hline $25 \ldots \ldots$ & 002358.1 & -720331 & 10.97 & $0.35 \pm 0.05$ & 122.2 & $\ldots$ & $\ldots$ & F260 & 11.94 & 1.61 & -24.4 \\
\hline $26 \ldots \ldots$ & 002359.0 & -720235 & 11.27 & $0.39 \pm 0.05$ & 124.3 & 1480 & 97.9 & $\mathrm{E} 370$ & 11.64 & 1.94 & -10.3 \\
\hline $27 \ldots \ldots$ & 002401.5 & -720301 & 11.36 & $0.38 \pm 0.05$ & 125.0 & $\ldots$ & $\cdots$ & $\mathrm{F} 276$ & 12.03 & 1.50 & -29.9 \\
\hline $28 \ldots \ldots$ & 002402.9 & -720811 & 11.66 & $0.34 \pm 0.06$ & 125.1 & $\ldots$ & $\ldots$ & E44 & 12.20 & 1.43 & -14.4 \\
\hline $29 \ldots .$. & 002404.2 & -720655 & 10.95 & $0.36 \pm 0.05$ & 127.4 & $\ldots$ & $\ldots$ & $\mathrm{F} 1$ & 11.90 & 1.51 & -25.5 \\
\hline $30 \ldots$. & 002406.5 & -720700 & 12.30 & $0.24 \pm 0.06$ & 124.2 & ... & $\therefore$. & F588 & 12.34 & 1.37 & -22.5 \\
\hline $31 \ldots$. & 002407.1 & -720330 & 10.71 & $0.39 \pm 0.05$ & 124.3 & $\ldots$ & $\ldots$ & $\mathrm{F} 290$ & 11.86 & 1.62 & -26.7 \\
\hline $32 \ldots$. & 002408.8 & -720259 & 10.95 & $0.33 \pm 0.05$ & 122.5 & $\ldots$ & $\ldots$ & F305 & 11.53 & 1.95 & -25.3 \\
\hline $33 \ldots$. & 002410.8 & -720701 & 12.00 & $0.29 \pm 0.06$ & 125.5 & $\ldots$ & $\ldots$ & F563 & 12.60 & 1.28 & $\ldots$ \\
\hline $34 \ldots$. & 002413.0 & -720650 & 12.25 & $0.32 \pm 0.06$ & 140.5 & $\ldots$ & $\ldots$ & F561 & 12.87 & 1.15 & $\ldots$ \\
\hline $35 \ldots \ldots$ & 002414.3 & -720635 & 11.94 & $0.32 \pm 0.06$ & 124.4 & $\ldots$ & $\ldots$ & F553 & 12.19 & 1.29 & -9.2 \\
\hline $36 \ldots \ldots$ & 002414.8 & -720445 & 10.50 & $0.38 \pm 0.04$ & 127.3 & $\ldots$ & $\ldots$ & $\ldots$ & $\ldots$ & $\ldots$ & $\ldots$ \\
\hline $37 \ldots \ldots$ & 002416.2 & -720132 & 11.45 & $0.37 \pm 0.06$ & 122.1 & $\ldots$ & $\ldots$ & E397 & 11.69 & 1.91 & -20.3 \\
\hline $38 \ldots \ldots$ & 002418.5 & -720444 & 10.11 & $0.41 \pm 0.03$ & 126.4 & $\ldots$ & $\ldots$ & $\ldots$ & $\ldots$ & $\ldots$ & $\ldots$ \\
\hline $39 \ldots \ldots$ & 002419.3 & -720519 & 11.20 & $0.48 \pm 0.05$ & 117.2 & ... & $\ldots$ & $\mathrm{F} 478$ & 11.93 & 1.58 & -36.2 \\
\hline $40 \ldots$. & 002420.3 & -720552 & 11.08 & $0.42 \pm 0.05$ & 122.3 & $\therefore$ & $\therefore$ & $\mathrm{F} 490$ & 12.08 & 1.44 & -19.8 \\
\hline $41 \ldots$. & 002420.5 & -720559 & 11.63 & $0.37 \pm 0.06$ & 118.6 & $\ldots$ & $\ldots$ & F507 & 12.17 & 1.42 & -9.6 \\
\hline $42 \ldots \ldots$ & 002421.0 & -720333 & 11.16 & $0.37 \pm 0.05$ & 125.2 & $\ldots$ & $\ldots$ & F338 & 11.80 & 1.70 & $\ldots$ \\
\hline $43 \ldots$. & 002423.4 & -720406 & 10.92 & $0.36 \pm 0.05$ & 122.1 & 1700 & 98.4 & $\ldots$ & ... & $\ldots$ & $\ldots$ \\
\hline $44 \ldots \ldots$ & 002424.8 & -720449 & 10.87 & $0.37 \pm 0.05$ & 117.5 & ... & $\ldots$ & F429 & 11.80 & 1.68 & -24.5 \\
\hline $45 \ldots \ldots$ & 002430.0 & -720432 & 11.55 & $0.42 \pm 0.06$ & 121.1 & 1774 & 56.6 & F400 & 11.82 & 1.69 & -27.8 \\
\hline $46 \ldots \ldots$ & 002430.7 & -720705 & 12.16 & $0.39 \pm 0.06$ & 126.7 & $\ldots$ & $\ldots$ & E630 & 12.88 & 1.20 & $\ldots$ \\
\hline $47 \ldots \ldots$ & 002430.9 & -720731 & 12.65 & $0.25 \pm 0.07$ & 127.2 & ... & $\ldots$ & E629 & 12.86 & 1.10 & ... \\
\hline $48 \ldots \ldots$ & 002433.9 & -720618 & 11.92 & $0.34 \pm 0.06$ & 120.2 & 1816 & 15.4 & E587 & 12.77 & 1.27 & -12.2 \\
\hline $49 \ldots$. & 002434.1 & -720513 & 12.07 & $0.43 \pm 0.06$ & 120.5 & $\ldots$ & $\ldots$ & F445 & 13.05 & 1.20 & $\ldots$ \\
\hline $50 \ldots \ldots$ & 002436.5 & -720540 & 11.34 & $0.43 \pm 0.05$ & 123.2 & $\ldots$ & $\ldots$ & E582 & 11.95 & 1.62 & -26.5 \\
\hline $51 \ldots$. & 002437.6 & -720511 & 11.73 & $0.31 \pm 0.06$ & 122.6 & 1862 & 0 & E647 & 12.78 & 1.10 & $\ldots$ \\
\hline $52 \ldots$. & 002441.6 & -720431 & 11.76 & $0.36 \pm 0.06$ & 122.8 & 1911 & 97.6 & E512 & 12.62 & 1.27 & -19.4 \\
\hline $53 \ldots$. & 002443.8 & -720723 & 11.62 & $0.43 \pm 0.06$ & 127.7 & 1940 & 96.4 & E620 & 12.00 & 1.59 & -24.3 \\
\hline
\end{tabular}

NotE.- - Units of right ascension are hours, minutes, and seconds, and units of declination are degrees, arcminutes, and arcseconds.

a Also listed as star 1222, with $\mathrm{MP}=0$. 

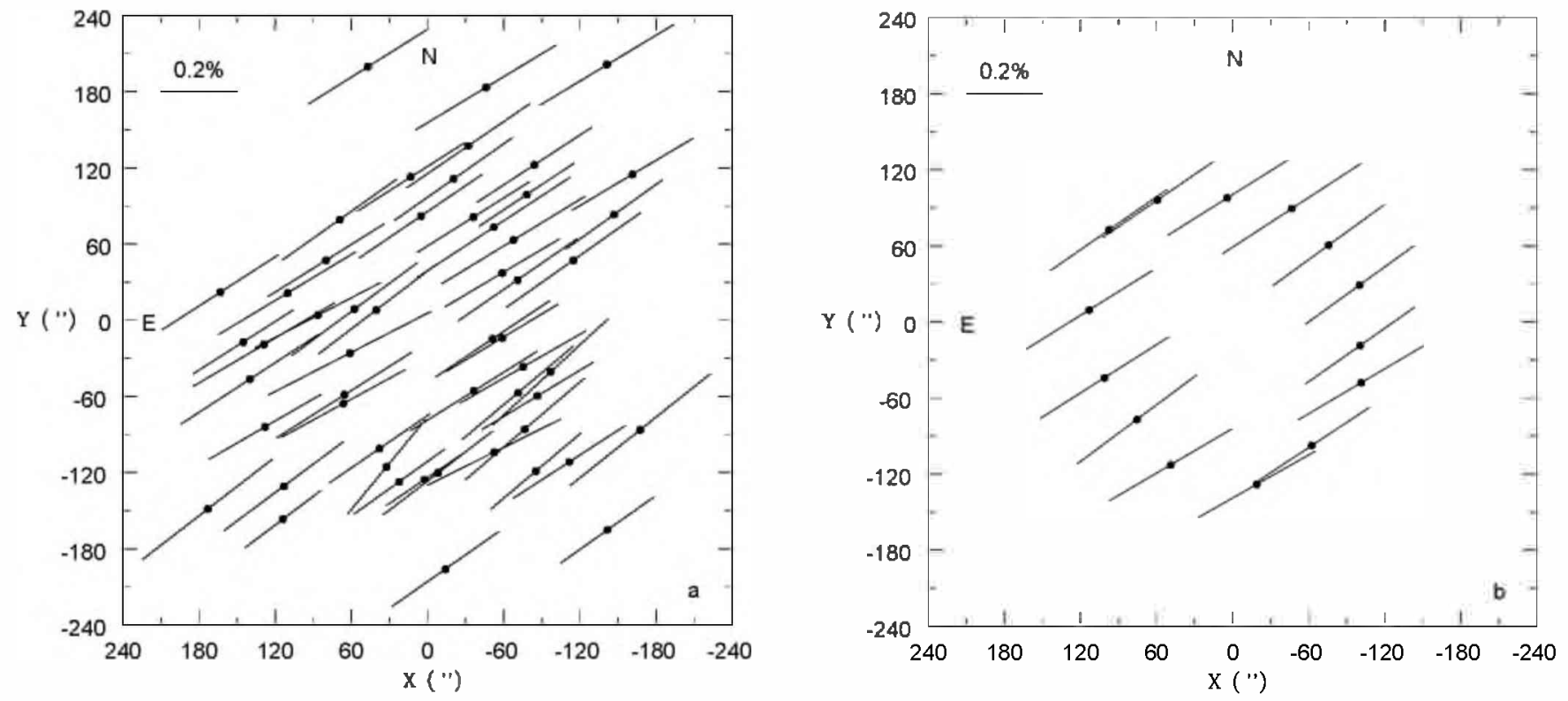

Fig. 2.-Visual polarization vectors for ( $a$ ) the 47 Tuc (nonvariable) red giants projected on the sky and ( $b$ ) the bright-star-free regions

\section{FOREGROUND POLARIZATION}

The detection of intrinsic polarization requires the removal of the foreground component arising from the interstellar dust along the line of sight to the cluster. Estimates of the color excess $E_{B-V}$ produced by this dust range from 0.03 to 0.04 (see Hesser et al. 1987).

In order to map the foreground polarization on a scale of few arcminutes, we first used the observations of the nonvariable stars listed in Table 1. The normalized Stokes parameters for these stars were approximated by a leastsquares fit to two planes, defined as

$$
U=a_{u} X+b_{u} Y+C_{u}, \quad Q=a_{q} X+b_{q} Y+C_{q},
$$

where $X$ and $Y$ are the rectangular coordinates of the stars (in arcseconds) in a system with the positive $X$-axis pointing toward the east, the positive $Y$-axis toward the north, and

TABLE 2

Visual Polarizations for Stars between $4^{\prime}$ and $20^{\prime}$ from the 47 Tucanae Nucleus

\begin{tabular}{crrrr}
\hline \hline $\begin{array}{c}X \\
\text { Star }^{\mathrm{a}}\end{array}$ & $\begin{array}{c}Y \\
(\operatorname{arcsec})\end{array}$ & $\begin{array}{c}Y \\
(\operatorname{arcsec})\end{array}$ & \multicolumn{1}{c}{$\begin{array}{c}P_{V} \\
(\%)\end{array}$} & $\begin{array}{c}\theta_{V} \\
(\mathrm{deg})\end{array}$ \\
\hline $1421 \ldots \ldots$. & -190.0 & -1060.0 & $0.53 \pm 0.05$ & 101.4 \\
$2620 \ldots \ldots$. & -555.9 & -167.2 & $0.43 \pm 0.05$ & 123.7 \\
$2705 \ldots \ldots$. & -492.0 & -73.8 & $0.50 \pm 0.07$ & 132.3 \\
$2758 \ldots \ldots$. & -423.1 & -373.9 & $0.42 \pm 0.04$ & 112.5 \\
$3708 \ldots \ldots$. & -546.1 & 64.0 & $0.39 \pm 0.05$ & 110.4 \\
$3730 \ldots \ldots$. & -418.2 & 167.4 & $0.44 \pm 0.05$ & 121.4 \\
$3736 \ldots \ldots$. & -433.0 & 196.9 & $0.44 \pm 0.06$ & 118.1 \\
$4715 \ldots \ldots$. & -275.5 & 260.8 & $0.41 \pm 0.04$ & 121.3 \\
$4728 \ldots \ldots$. & 50.0 & 541.3 & $0.48 \pm 0.05$ & 111.5 \\
$4729 \ldots \ldots$. & 50.0 & 492.1 & $0.37 \pm 0.06$ & 117.9 \\
$5312 \ldots \ldots$. & 806.9 & 885.7 & $0.47 \pm 0.06$ & 118.0 \\
$5529 \ldots \ldots$. & 561.0 & 659.4 & $0.46 \pm 0.05$ & 114.7 \\
$5622 \ldots \ldots$. & 226.3 & 551.1 & $0.40 \pm 0.05$ & 104.9 \\
$8756 \ldots \ldots$. & 255.8 & -295.2 & $0.28 \pm 0.07$ & 121.6 \\
\hline
\end{tabular}

a Identification number from Lee 1977. the origin at the cluster center. The $a$ - and $b$-coefficients are then percentages per arcsecond and the $C$-coefficients are percentages. The resulting coefficients and associated errors are

$$
\begin{array}{cl}
a_{u}=(-7.7 \pm 2.5) \times 10^{-6}, & a_{q}=(-1.22 \pm 1.5) \times 10^{-4}, \\
b_{u}=(-4.95 \pm 2.0) \times 10^{-6}, & b_{q}=(-2.15 \pm 0.5) \times 10^{-4}, \\
C_{u}=-0.34 \pm 0.02, & C_{q}=-0.14 \pm 0.02,
\end{array}
$$

and they show a mild trend of the parameter $U$ with both $X$ and $Y$, while $Q$ exhibits a detectable positional dependence mainly with the $Y$-coordinate. A comparison between the polarization predicted by the plane fitting (at the position of each star) and a smoothed average of the observed polarizations as a function of position angle (measured at the center of the cluster) is depicted in Figure 3. The smoothed-average polarizations were obtained by computing the mean $U$ - and $Q$-values within sectors $45^{\circ}$ wide and adopting a step of 22.5 . This figure shows that the plane fit is a good overall representation of the polarization variation within $4^{\prime}$ from

\begin{tabular}{|c|c|c|c|c|}
\hline Star $^{\mathrm{a}}$ & $\begin{array}{c}P_{V} \\
(\%)\end{array}$ & $\begin{array}{c}\theta_{V^{r}} \\
(\%)\end{array}$ & $\begin{array}{c}U \\
(\%)\end{array}$ & $\underset{(\%)}{Q}$ \\
\hline V1 ........... & $0.36 \pm 0.06$ & 99.0 & -0.11 & -0.34 \\
\hline $\mathrm{V} 2 \ldots \ldots \ldots$ & $0.06 \pm 0.05$ & 45.0 & 0.06 & 0.00 \\
\hline V3 ............. & $0.42 \pm 0.05$ & 105.1 & -0.21 & -0.36 \\
\hline V4 ............. & $0.47 \pm 0.08$ & 130.7 & -0.46 & -0.07 \\
\hline $\mathrm{V} 4 \ldots \ldots \ldots$ & $0.23 \pm 0.04$ & 52.6 & 0.22 & -0.06 \\
\hline V6 .............. & $0.36 \pm 0.06$ & 135.8 & -0.36 & 0.01 \\
\hline V8 ............. & $0.47 \pm 0.04$ & 1.8 & 0.03 & 0.47 \\
\hline V10 ......... & $0.31 \pm 0.04$ & 120.5 & -0.27 & -0.15 \\
\hline V11 ......... & $0.47 \pm 0.06$ & 135.6 & -0.47 & 0.01 \\
\hline V13 ......... & $2.37 \pm 0.10$ & 123.9 & -2.20 & -0.90 \\
\hline
\end{tabular}

TABLE 3

Visual Polarizations for Variable StaRs in 47 Tucanae

${ }^{a}$ Identification from Sawyer Hogg 1973. 
TABLE 4

Visual Polarization for the Bright-Star-free Regions IN THE FiELd OF 47 TUCANAE

\begin{tabular}{cccccrr}
\hline \hline & $\begin{array}{c}\text { R.A. } \\
\text { No. }\end{array}$ & $\begin{array}{c}\text { Decl. } \\
(\mathrm{J} 2000)\end{array}$ & $\begin{array}{c}V_{17^{\prime \prime}} \\
(\mathrm{mag})\end{array}$ & $\begin{array}{c}P_{I^{r}} \\
(\%)\end{array}$ & $\begin{array}{c}\theta_{V} \\
(\mathrm{deg})\end{array}$ & $\begin{array}{r}\text { P.A. } \\
(\mathrm{deg})\end{array}$ \\
\hline $1 \ldots \ldots \ldots$. & 002401.84 & -720701.8 & 12.78 & $0.35 \pm 0.06$ & 119.5 & 188.4 \\
$2 \ldots \ldots \ldots$. & 002352.40 & -720631.0 & 11.78 & $0.36 \pm 0.04$ & 123.5 & 212.5 \\
$3 \ldots \ldots \ldots$. & 002343.94 & -720541.3 & 12.23 & $0.38 \pm 0.05$ & 120.7 & 244.6 \\
$4 \ldots \ldots \ldots$. & 002344.13 & -720511.8 & 11.89 & $0.35 \pm 0.04$ & 125.0 & 259.3 \\
$5 \ldots \ldots \ldots$. & 002344.23 & -720423.8 & 11.67 & $0.35 \pm 0.04$ & 125.8 & 286.1 \\
$6 \ldots \ldots \ldots$. & 002349.56 & -720352.4 & 12.03 & $0.36 \pm 0.04$ & 126.0 & 308.6 \\
$7 \ldots \ldots \ldots$. & 002355.85 & -720323.4 & 11.99 & $0.43 \pm 0.04$ & 123.2 & 331.2 \\
$8 \ldots \ldots \ldots$. & 002406.92 & -720315.0 & 11.83 & $0.37 \pm 0.03$ & 122.1 & 2.6 \\
$9 \ldots \ldots \ldots$. & 002418.85 & -720316.7 & 11.67 & $0.35 \pm 0.04$ & 124.2 & 31.8 \\
$10 \ldots \ldots \ldots$. & 002417.14 & -720340.6 & 11.86 & $0.37 \pm 0.04$ & 124.8 & 53.5 \\
$11 \ldots \ldots \ldots$. & 002430.56 & -720443.9 & 11.49 & $0.39 \pm 0.03$ & 122.0 & 85.4 \\
$12 \ldots \ldots \ldots$. & 002427.96 & -720538.0 & 12.72 & $0.40 \pm 0.06$ & 122.4 & 113.8 \\
$13 \ldots \ldots \ldots$. & 002422.37 & -720610.7 & 12.39 & $0.39 \pm 0.05$ & 126.7 & 135.8 \\
$14 \ldots \ldots \ldots$. & 002416.61 & -720646.5 & 12.41 & $0.37 \pm 0.06$ & 120.3 & 156.5 \\
\hline
\end{tabular}

the cluster center both in amplitude and in angle. In turn, the $C_{u}$ and $C_{q}$ lead to $P_{V}=0.37 \% \pm 0.02 \%$ and $\theta_{V}=123.8$ for the interstellar polarization at the cluster center, in very good agreement with the (unfiltered) polarimetry by Mathewson \& Ford (1970), who obtained $P=0.36 \% \pm 0.09 \%$ and $\theta=123 \%$.

If a color excess $E_{B-V}=0.04$ is adopted for the interstellar reddening toward $47 \mathrm{Tuc}$, and taking into account the relation between polarization efficiency and color excess derived by Serkowski, Mathewson, \& Ford (1975), $P_{V}=9.0 E_{B-V}$, then the estimated polarization for the cluster center is compatible with a situation of maximum grain alignment and, thus, maximum polarization efficiency (i.e., degree of polarization per color excess or extinction in magnitudes). We note that the IRAS calibrated $E_{B-V}$ map by Schlegel, Finkbeiner, \& Davis (1998) predicts a somewhat smaller color excess, $E_{B-V}=0.03$, at the position of 47 Tuc's center. This map shows a very uniform color excess on an angular scale of 4 ' from the cluster center, that is, the polarization variation seems more associated with a change in polarization efficiency than with varying foreground extinction.

The polarization behavior within $4^{\prime}$ of the cluster center can be compared with that of the stars listed in Table 2 (located between $4^{\prime}$ and $20^{\prime}$ from the center). In this case, the relatively small number of member stars and the somewhat uneven distribution on the sky prevent a treatment similar

TABLE 5

UBVRI Polarizations For the NUCleus of 47 TuCANAE

\begin{tabular}{cccc}
\hline \hline Filter & $\begin{array}{c}\lambda \\
(\mathrm{nm})\end{array}$ & $\begin{array}{c}P_{\lambda} \\
(\%)\end{array}$ & $\begin{array}{c}\theta_{\lambda} \\
(\mathrm{deg})\end{array}$ \\
\hline$U:$ & & & \\
$17^{\prime \prime} \ldots \ldots$. & 360 & $0.44 \pm 0.11$ & $127.4 \pm 2.5$ \\
$33^{\prime \prime} \ldots \ldots$. & 360 & $0.72 \pm 0.06$ & $118.6 \pm 2.5$ \\
$45^{\prime \prime} \ldots \ldots$. & 360 & $0.48 \pm 0.06$ & $116.8 \pm 2.0$ \\
$B \ldots \ldots \ldots .$. & 430 & $0.46 \pm 0.02$ & $125.6 \pm 1.0$ \\
${ }^{\prime} \ldots \ldots \ldots \ldots$. & 550 & $0.36 \pm 0.01$ & $124.5 \pm 0.5$ \\
$R \ldots \ldots \ldots \ldots$. & 630 & $0.33 \pm 0.01$ & $124.4 \pm 0.5$ \\
$1 \ldots \ldots \ldots \ldots .$. & 780 & $0.29 \pm 0.03$ & $123.8 \pm 1.5$ \\
\hline
\end{tabular}

to that of the inner field. However, as shown in Figure 4, the behavior of $P_{\mathrm{I}}$ for stars in this sample, and that of the inner field stars, is compatible with an overall variation of the polarization along the north-south direction. Thus, the observed variation within $4^{\prime}$ of the cluster center does not seem to be a feature just connected with the cluster but, rather, a consequence of a variation of the polarization on a larger angular scale on the sky.

Figure 4 does not include the star L1421 (listed in Table 2), since, as mentioned in $\S 2$, this object appears to be associated with an elongated infrared structure and, after removing the interstellar component, shows an intrinsic polarization $P_{V}=0.38 \%$ and $\theta_{V}=80.0$.

Figure 5, in turn, shows the variation of the polarization observed in the bright-star-free regions, as a function of their position angle, compared with the smooth average polarization of the nonvariable stars. The very good agreement, in this case, also lends support to the idea that most of the observed variation of the polarization is due to the foreground interstellar component.

\section{RESIDUAL POLARIZATIONS}

The residual polarizations, after subtracting the foreground component defined by the parameters discussed in the previous section, are shown on the sky plane both for the inner field stars and for the star-free regions in Figure 6 and on the Stokes plane in Figure 7. The main features in these diagrams are that (1) most of the residual polarizations seem consistent with observational errors, and practically, no star exhibits values exceeding the $3 \sigma_{P_{V}}$ level; and (2) there is no systematic angular pattern, either for the star or for the star-free region polarizations. From these results, we conclude that intrinsic polarizations associated with dusty shells are not detectable in most of the nonvariable stars.

Even though these residual polarizations are not large compared with the errors of the observations, a plot of these values as a function of the $Y$-coordinate (along the northsouth direction), displayed in Figure 8, shows a systematic trend in the sense that the larger polarizations are located toward the south of the cluster center. Eight (out of nine) 

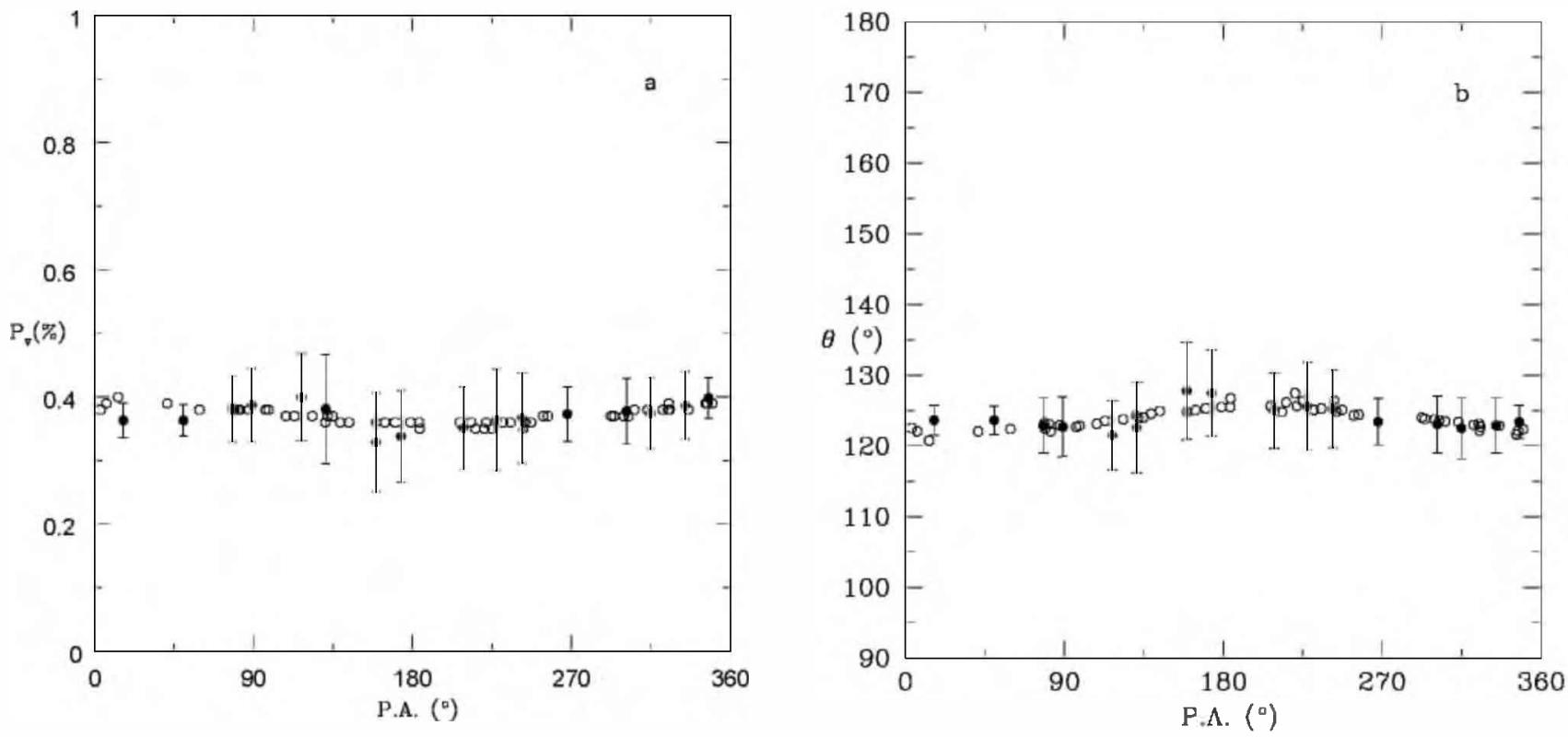

FIG. 3. Smoothed (a) polarization $\nu_{V}$ and $(b)$ polarization angle $\theta_{V}$ for the red giants, compared with the expected polarizations obtained from the $U$ and $Q$ plane fittings (open circles) as a function of position angle measured at the cluster center. The bars represent the dispersion of the mean polarization values.

stars with residual polarizations at the $2 \sigma_{P_{V}}$ level or higher appear in that area. This trend is also seen in the two southernmost star-free regions (also plotted in Fig. 8). Although this effect is marginal and deserves higher precision polarimetry in order to be confirmed, it could be an indication of the presence of small amounts of scattered light. As the polarimeter aperture includes an important fraction of background light, the nature of the observed polarization, that is, whether the residual polarizations originate from the stars or from the background light, is not clear. However, as most of the bright-star-free regions do not show detectable polarization excesses, we are inclined to believe that the residual polarizations could arise in low optical depth dust

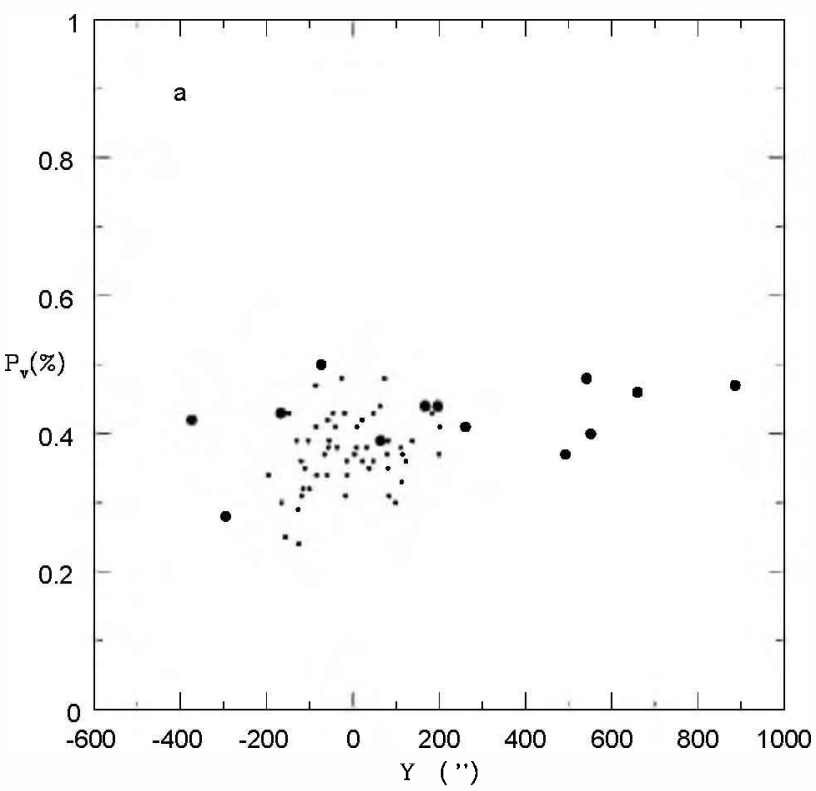

that is locally illuminated by (but not necessarily originating from) some stars in the southern region. In this case, and assuming that the background light within the diaphragm is only polarized by the foreground dust along the line of sight to the cluster, the residual polarizations should be increased by a factor of $10^{0.4 \delta m}$, where $\delta m$ is the difference between the star and the total $V$ magnitude listed in Table 1. In the most extreme cases, such a correction leads to polarization excesses as large as $0.35 \%$.

The fact that the proper motion of the cluster in its own local standard of rest, as shown in Table 3 of Krockenberger \& Grindlay (1995), points toward the north, may provide some basis to speculate about the nature of the observed

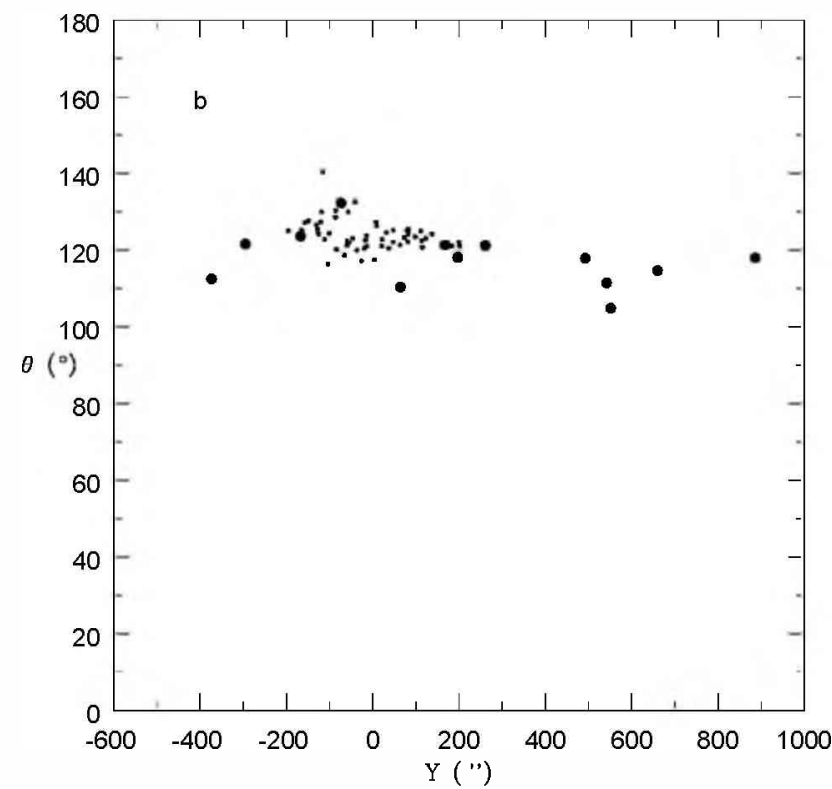

Fig. 4.- (a) Polarization $P_{V}$ vs. $Y$-coordinate (south to north) and (b) polarization angle $\theta$ vs. $Y$ for the outer 47 Tuc field (large circles) and for the inner cluster field (dots). 


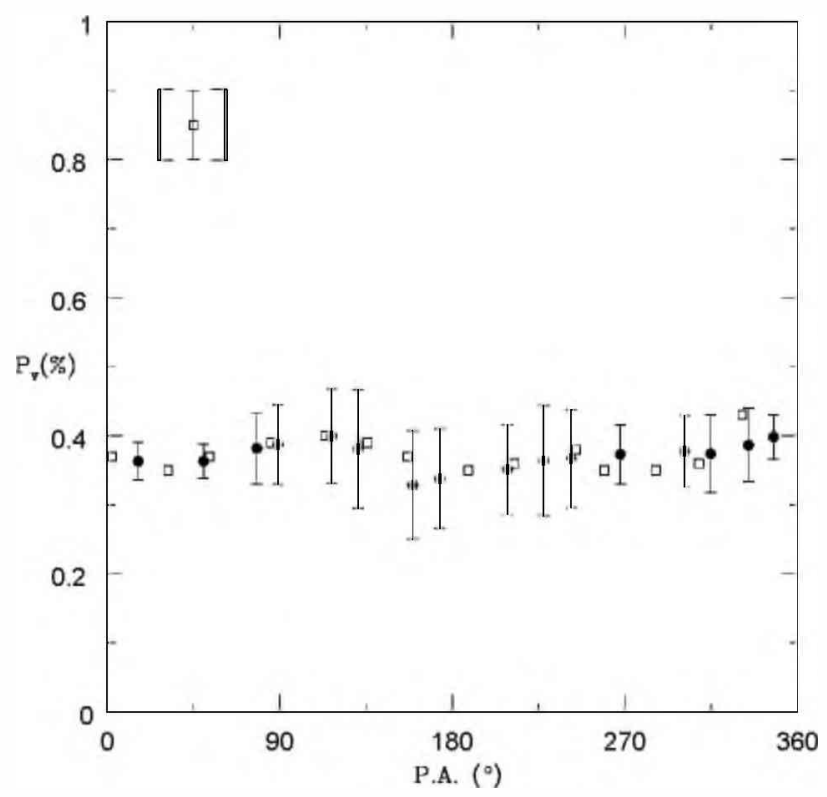

FIG. 5. Comparison of the smoothed star polarizations with those corresponding to the bright-star-free regions (squares) as a function of position angle. Polarization samples were taken within $45^{\circ}$ sectors moved in 22.5 steps. The bars represent the dispersion of the mean polarization values. The bar at the upper left is representative of the errors for the bright-star-free regions.

spatial asymmetry of the residual polarizations seen in the opposite direction (see $\S 10$ ).

\section{VARIABLE STARS}

The observed $U$ and $Q$ parameters for the group of nine red luminous variables in our sample are displayed in Figure 9. This figure also includes a circle with a $2.5 \sigma_{P_{V}}$ radius, which contains practically all the nonvariable stars (also plotted). The circle is centered at the $U$ - and $Q$-values that are representative of the interstellar polarization (parameters $C_{u}$ and $C_{q}$ in $\S 4$ ). Except for the variable star V10, all the remaining ones fall outside the error circle and thus exhibit a significant excess or intrinsic polarization. For seven of these stars, the excess polarizations (not corrected for the background dilution effect mentioned in the preceding section) range from $0.15 \%$ to $0.70 \%$ and show no preferential alignments (but see the case of the comparatively highly polarized V13, below).

The fact that most of the variable stars exhibit intrinsic polarization agrees with a previous tentative conclusion by Origlia et al. (1997), who point out that " in the circumstellar environments of V1, V2, V3 and V4 we have some indication of intrinsic polarization." In the particular case of $\mathrm{V} 1$, these authors also report a detection at $10 \mu \mathrm{m}$, attributed to dust emission. The presence of dust around V3, in turn, is supported by the high $12 \mu \mathrm{m}$-to $2 \mu \mathrm{m}$ flux ratio found by Ramdani \& Jorissen (2001). These last authors also mention a marginal detection of dust emission around V11 based on the same flux ratio.

The variable star V13 is an interesting object (although its membership into the cluster remains to be confirmed). Its intrinsic polarization, after removing the foreground interstellar component, reaches $P_{V}=2.0 \% \pm 0.15 \%$ with a polarization angle $\theta=123^{\circ} 9 \pm 3^{\circ}$, which is remarkably similar to that of the foreground polarization itself. This coincidence requires a further analysis in order to clarify whether it happens just by chance or, alternatively, if the alignment mechanism of the foreground dust and the mechanism that controls the circumstellar polarization in V13 are coupled in some way. Since this latter polarization is thought to arise from scattering (as supported by preliminary $B$-band observations that show an increase of the polarization toward shorter wavelengths in V13), while the foreground dust polarization is associated with large-scale magnetic fields, the physics behind this hypothetical coupling is not clear. If dust alignment by these magnetic fields is responsible for the observed polarization, then, in the case

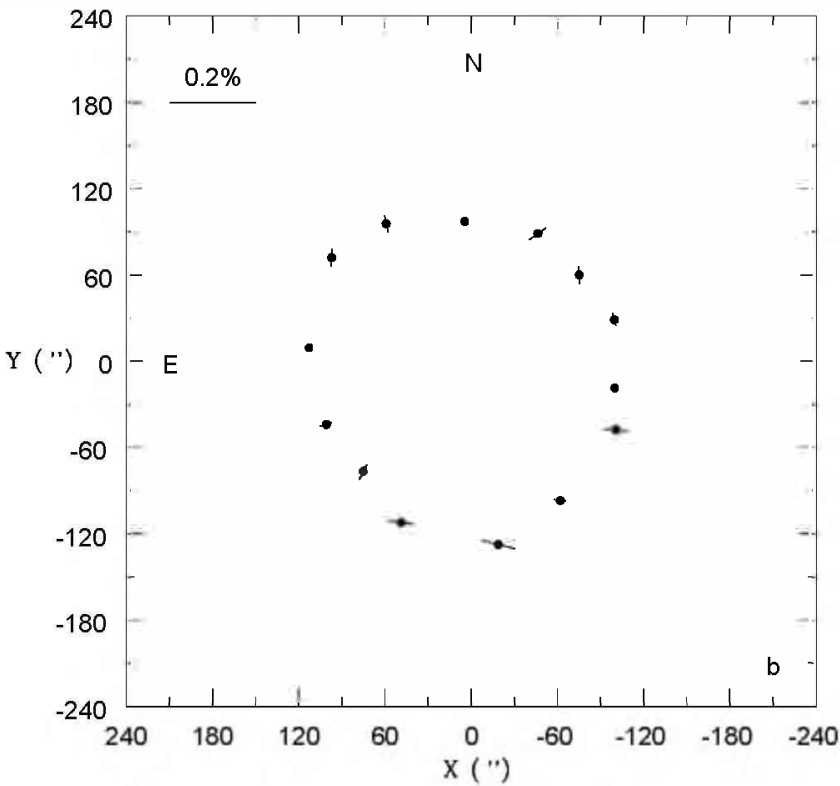

Fig. 6.-Residual polarizations (i.e., after removing the foreground interstellar component) for ( $a$ ) the red giants and (b) the star-free regions projected on the sky. 


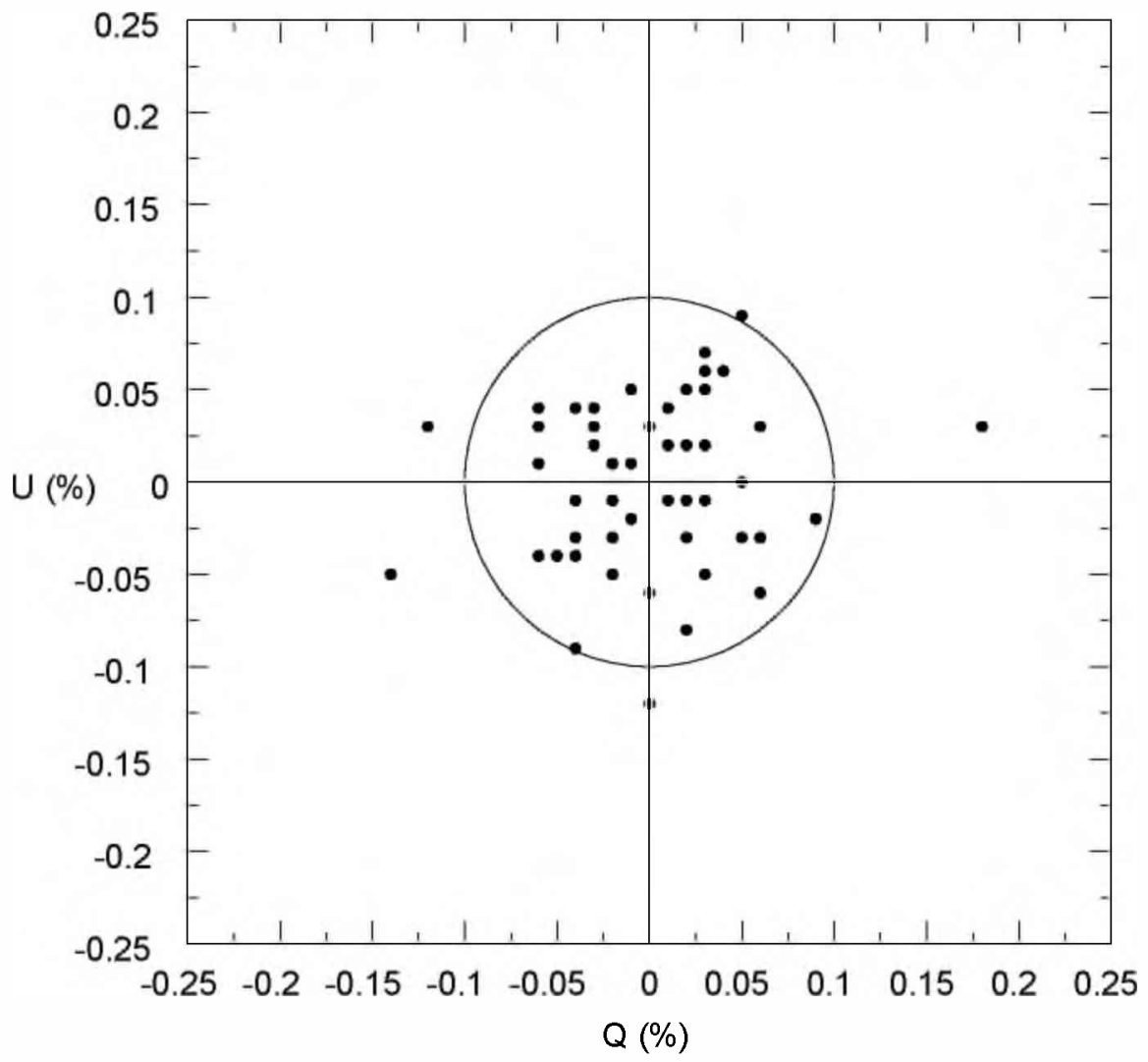

Fig. 7. $-U$ vs. $Q$ Stokes plane for the nonvariable red giants after removing the interstellar polarization. The circle has a radius set by $2.5 \sigma_{P_{V}}$.

of maximum polarization efficiency, a color excess $E_{B-V}=0.25$ would be required. This excess implies an interstellar reddening some 7 times higher than that estimated for the 47 Tuc field.

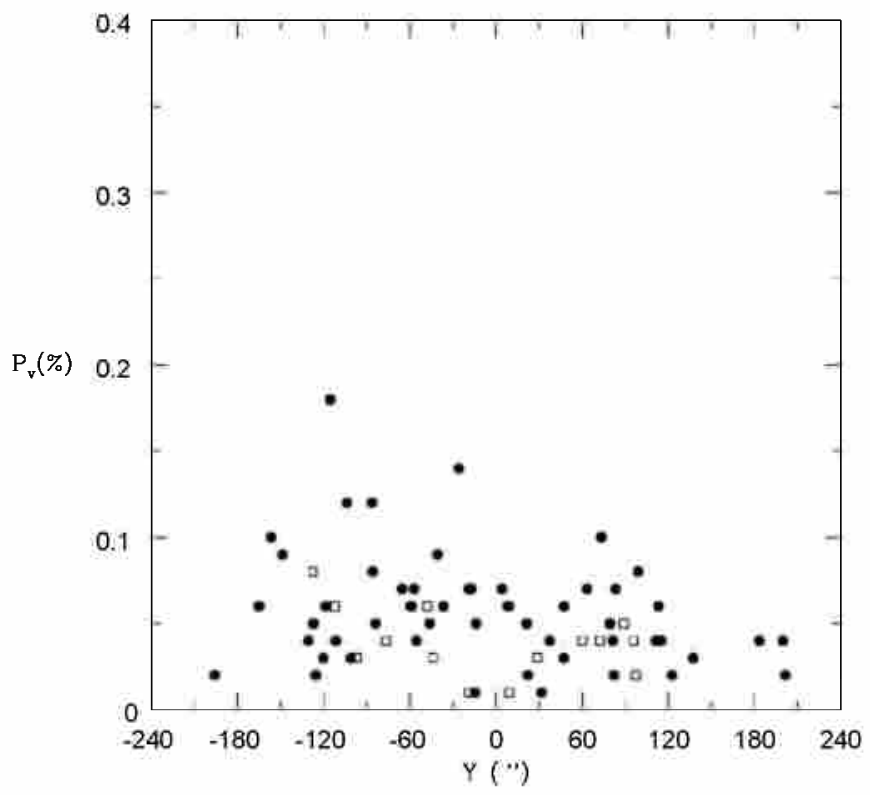

Fig. 8. - Residual polarizations for the red giants (circles) and for the star-free regions (squares) as a function of $Y$-coordinate (south to north). An increasing trend is seen toward the south of the cluster.

\section{MULTICOLOR POLARIZATION OBSERVATIONS OF THE 47 TUCANAE NUCLEUS}

The UBVRI polarization observations of the cluster nucleus, listed in Table 5, are depicted as a function of wavelength in Figure 10. Usually, the wavelength dependence of the interstellar polarization can be adequately fitted with a Serkowski (1973) curve,

$$
P_{\lambda} / P_{\lambda_{\text {Tax }}}=\exp \left\{-K\left[\ln \left(\lambda / \lambda_{\max }\right)\right]^{2}\right\},
$$

which involves three parameters: $\lambda_{\max }, P_{\lambda_{\max }}$, and $K$. Later discussions by Wilking, Lebofsky, \& Rieke (1982) and Whittet et al. (1992) have shown the existence of a linear correlation between $K$ and $\lambda_{\max }$. In most cases, the interstellar polarization exhibits a peak at a wavelength close to 0.55 $\mu \mathrm{m}$, which is consistent with a selective extinction ratio $R \sim 3.0$ (Serkowski et al. 1975), a behavior that stands in contrast with the fact that the nucleus of 47 Tuc shows a monotonic increase of the polarization toward shorter wavelengths. This situation cannot be considered, in principle, as an anomalous case, as shown by some objects observed by Whittet et al. (1992; see their Fig. 3) that have maximum polarization wavelengths as short as $\sim 0.35 \mu \mathrm{m}$. The assumption that the interstellar polarization curve for the 47 Tuc nucleus peaks in the $U$ band would imply $K=0.61$ according to the $K$ versus $\lambda_{\max }$ relation given by Whittet et al. (1992). However, such a curve, normalized to $P_{V}=0.36 \%$, provides a poor representation of the observed data, as shown by Figure 10, where another fit, just considering the $V R I$ values, is also shown. Moreover, the $U$-band observations through the $33^{\prime \prime}$ and $45^{\prime \prime}$ diaphragms exhibit a 


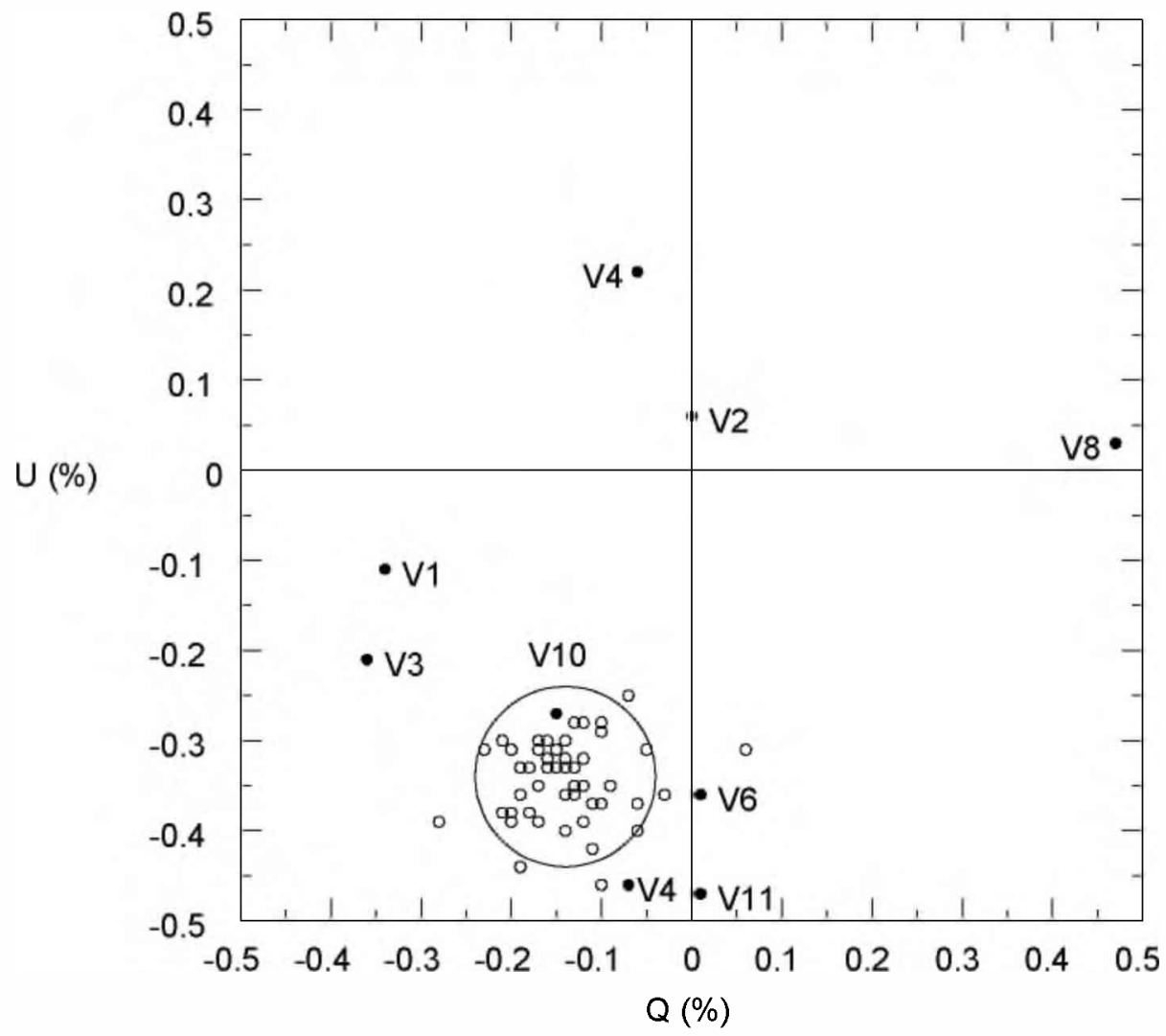

Fig. 9.-Stokes $U$ vs. $Q$ plane for the variable stars (filled circles). The observations corresponding to V4 are indicated in a separate way. Nonvariable red giants are shown as open circles. The large circle has a $2.5 \sigma_{P_{V}}$ radius and is centered at the $(U, O)$-values corresponding to the mean interstellar polarization along the line of sight toward the cluster. V13 falls outside the limits of the figure.

significant variation in polarization amplitude and a different angle compared with those observed for the other filters. These two features suggest that the observed polarization cannot be entirely explained as a result of an interstellar

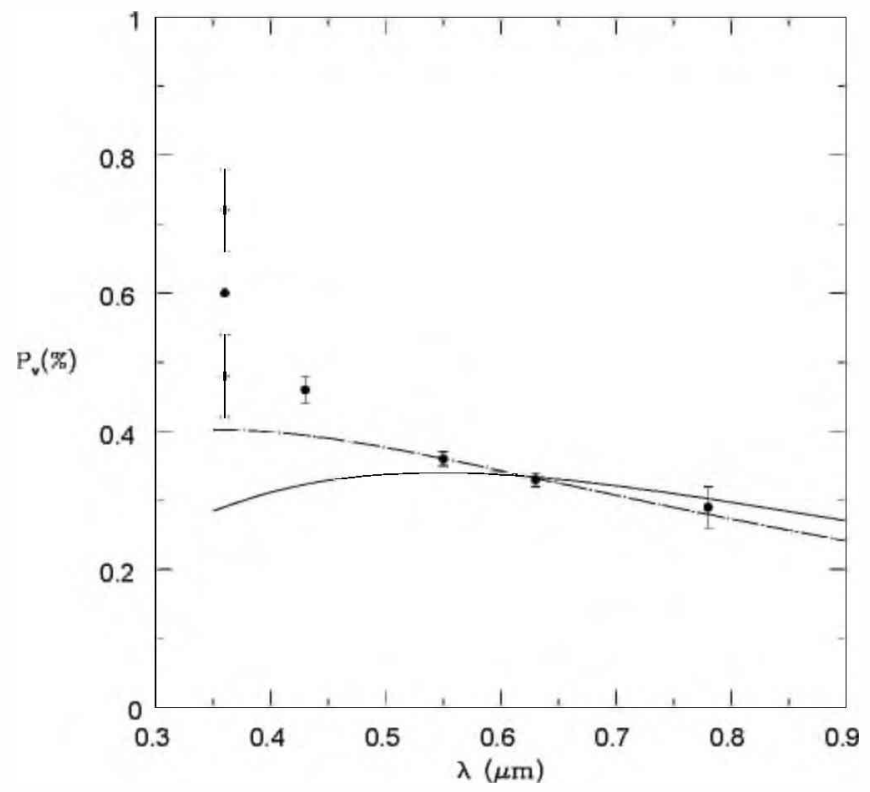

FIG. 10.-Linear polarization observed in the 47 Tuc nucleus as a function of wavelength of the $U B V R I$ bands. Three different values are given for the $U$ filter, corresponding to the $33^{\prime \prime}$ and $45^{\prime \prime}$ diaphragms and to their average. The lines correspond to two different Serkowski-law fits, with $\lambda_{\text {min }}$ of $0.55 \mu \mathrm{m}$ (solid line) and $0.36 \mu \mathrm{m}$ (dot-dashed line) . component typified by somewhat extreme values of $K$ and $\lambda_{\max }$.

We note that the observed visual polarization for the nucleus agrees, within the errors, with the estimated foreground polarization discussed in $\S 3$, thus suggesting that the excess polarization in the $V$ band, if any, is very small.

\section{A TENTATIVE EXPLANATION FOR THE EXCESS POLARIZATION IN THE 47 TUCANAE NUCLEUS}

The bright (and blue) post- $A$ GB star referred to as BS or UIT 7 (see $\S 3.1$ ) is located some $48^{\prime \prime}$ from the cluster center and appears to be a suitable candidate for consideration as the origin of the excess polarization in the nucleus, for two reasons:

1. The angle of the excess polarization, after removing the ultraviolet interstellar polarization expected from a Serkowski curve, is close to "tangential" (i.e., perpendicular to the line joining the star and the cluster center; see below), as would be expected if some dust in the nuclear region were asymmetrically illuminated by the star.

2. The ratio of the stellar to nuclear flux, as a function of wavelength, increases steeply toward the ultraviolet, thus decreasing the dilution effect produced by the light of the nucleus within a given aperture. This effect would be reflected, as observed, as an increase of the excess polarization in $U$ and, to a lesser extent, at longer wavelengths, where that flux ratio decreases. 
We quantify this polarization excess first by adopting the average of the polarization measures through the $33^{\prime \prime}$ and $45^{\prime \prime}$ diaphragms, $P_{U}=0.60 \%$ and $\theta=117.9$, and second by removing the ultraviolet interstellar polarization. For this component, a maximum polarization wavelength of 0.36 $\mu \mathrm{m}$ (from Fig. 10) or $0.55 \mu \mathrm{m}$ (compatible with a more "normal" behavior), with $P_{V}=0.36 \%$ and $\theta_{V}=123.8$, leads to an excess polarization in the $U$ band of $0.23 \%$ or $0.30 \%$, with angles $\theta_{U}=118^{\circ} .9$ and $\theta_{U}=113.7$, respectively. These polarization angles lie less than $20^{\circ}$ from the angle of the direction perpendicular to the line joining the star and the cluster nucleus $\left(\sim 132^{\circ}\right)$, that is, the polarization angle expected if dust were effectively illuminated by the post-AGB star.

A quantitative estimate of the dust optical depth required to give rise to such excess polarization requires the following:

1. Knowledge of the spectral energy distributions for both the star and the cluster nucleus. The spectral type of UIT 7 is B8 III with an apparent visual magnitude $V=10.73$, according to Table 1 in O'Connell et al. (1997), implying a temperature $T=13,450 \mathrm{~K}$ and intrinsic colors $(U-B)_{0}=-0.44$ and $(B-V)_{0}=-0.14$ (from Cramer 1984). Alternatively, Dixon et al. (1995) obtained a considerably lower temperature, $T=10,500 \mathrm{~K}$, corresponding to $(U-B)_{0}=-0.20$ and $(B-V)_{0}=-0.01$ (Flower 1996). As a compromise we take the average of these indexes, $(U-B)_{0}=-0.32$ and $(B-V)_{0}=-0.08$, as representative of the intrinsic colors of UIT 7. In turn, adopting a color excess $E_{B-V}=0.04$ toward the cluster and a selective extinction ratio $R=3.0$ leads to interstellar extinction-corrected magnitudes $U_{0}=10.21, B_{0}=10.53$, and $V_{0}=10.61$ for the star.

For the nucleus, we adopted the reddening-corrected colors given by Reed, Hesser, \& Shawl (1988), $(U-B)_{0}=0.31$ and $(B-V)_{0}=0.82$. Within an aperture of $33^{\prime \prime}$ we obtain $V \sim 7.50$, and thus $U_{0}=8.51, B_{0}=8.20$, and $V_{0}=7.38$.

A comparison of the magnitudes of the star and the nucleus shows a rapid increase of the relative brightness of UIT 7 toward short wavelengths, a fact that is dramatically shown by Figure 1 of O'Connell et al. (1997), where the far-ultraviolet luminosity of the star exceeds the integrated brightness of the cluster within the visual half-light radius $\left(174^{\prime \prime}\right)$.

2. Adoption of a relative geometry involving the cluster nucleus, the star, and the observer. The star UIT 7 appears at a projected distance on the sky of $48^{\prime \prime}$ from the cluster center. As we have no indication about the relative position of the star and the cluster nucleus along the line of sight, we leave this quantity as a free parameter in our calculation.

3. Adoption of parameters for the dust grains such as size, albedo, and phase function. The fact that the excess polarization increases rapidly toward the ultraviolet and favors a $\lambda^{-4}$ type of dependence rather than a $\lambda^{-1}$ law, which characterizes " normal" interstellar grains, indicates that the dust scatterers might be small compared with the light wavelength. As a first approach, then, we adopt highly reflective particles (i.e., unity albedo) and a Rayleigh-like phase function.

4. The size, spatial dust distribution, and optical depth of the cloud. The behavior of the ultraviolet polarization, which increases from the $17^{\prime \prime}$ diaphragm toward a maximum at $33^{\prime \prime}$ and then decreases at $45^{\prime \prime}$, suggests that most of the polarization arises from within the second aperture, and thus we adopt an indicative cloud radius of 16 ".5 (on the sky). The spatial dust density was represented with a function consistent with a King profile with $r_{c}=24^{\prime \prime}$, truncated at the indicative radius mentioned above, and an optical depth (through the whole cloud diameter) $\tau_{U}=0.5$. This value corresponds to a weighted optical depth of $\sim 0.3$ for the whole cloud, which may be somewhat large for an optically thin approach and would require depolarization corrections in a more rigorous model.

Figure 11 depicts the results, obtained with all the mentioned assumptions, for the ratio of scattered light arising in the illuminating star UIT 7 to that of the integrated stellar light of the nucleus in the $U B V$ bands, as a function of the "depth" coordinate, that is, the position of the star along the line of sight in core radius units (Fig. 11a), as well as the resulting polarization from a single-scattering approach (and equations from van de Hulst 1957; Fig. 11b).

This figure indicates that the order of the observed excess polarization can be accounted for by a relatively low optical depth dust cloud centered at the cluster nucleus and illuminated by the star UIT 7 . We note that if this cloud is mixed with the stars and also illuminated by the cluster radiation field, then its effect on the integrated cluster light profile will be considerably decreased. The adoption of models like those discussed by Witt, Thronson, \& Capuano (1992) shows that the central profile brightness would be dimmed by between 0.16 and $0.25 \mathrm{mag}$ in the $U$ band (for an isotropic-scattering or a Henyey-Greenstein phase function typified by an anisotropy factor $g=0.7$ ) and by less than $0.1 \mathrm{mag}$ in $V$.

The estimate of the mass associated with such a cloud depends on the nature of the dust grains. Following Kanagy $\&$ Wyatt (1978) and adopting a cloud radius of $0.36 \mathrm{pc}$, corresponding to an angular radius of 16.5 at a distance of 4.7 kpc (from Hesser et al. 1987),

$$
M_{d}=0.18 \rho \tau Q^{-1} r_{g} M_{\odot},
$$

where $\tau$ is the average optical depth of the spherical cloud, $\rho$ is the density (in $\mathrm{g} \mathrm{cm}^{-3}$ ) of a dust grain, $Q$ is its extinction efficiency, and $r_{g}$ is its radius in microns. For example, the adoption of astronomical silicate grains (Draine \& Lee 1984 ) with radii on the order of a few hundred angstroms and $\tau_{U}=0.3$ leads to a total dust mass of $\sim 0.05 M_{\odot}$. This is well above the estimates from far-infrared observations (see $\S 10$ ) and includes the uncertainty connected to the fact that interstellar grains may not be an adequate approximation to circumstellar dust (if this is the origin of the dust particles producing polarization).

\section{CLOUD CANDIDATES IN THE 47 TUCANAE NUCLEAR REGION}

An inspection of Hubble Space Telescope (HST) archival multicolor images reveals the presence of several dark patches within $30^{\prime \prime}$ of the cluster nucleus. One of these patches, which exhibits a triangular shape $\left(5^{\prime \prime} \times 3^{\prime \prime}\right)$, appears $12^{\prime \prime}$ from the cluster center and at a position angle of $120^{\circ}$. Two polarimetric measures were obtained at the position of this dark patch through a $5^{\prime \prime}$ aperture diaphragm during the 2000 and 2001 runs. These observations were made with the $V$ filter and integration times of $300 \mathrm{~s}$ each, yielding $P_{V}=0.42 \% \pm 0.02 \%$ and $\theta=123.2 \pm 2^{\circ}$. This polarization is larger, by some $20 \%$, than that of the nucleus 


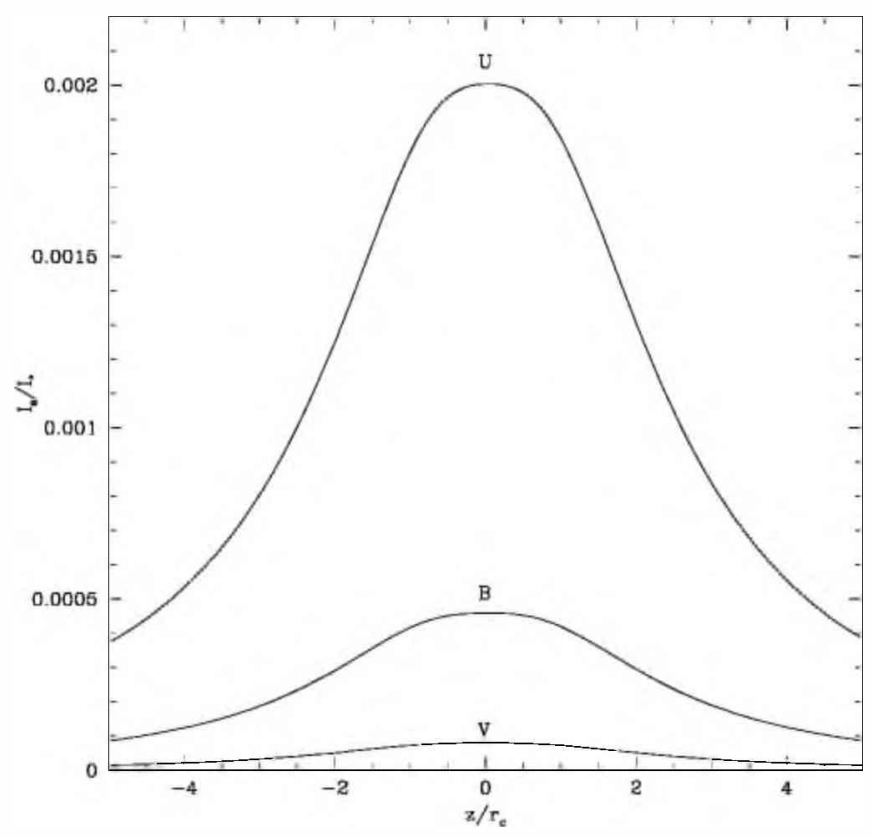

FIG. $11 a$

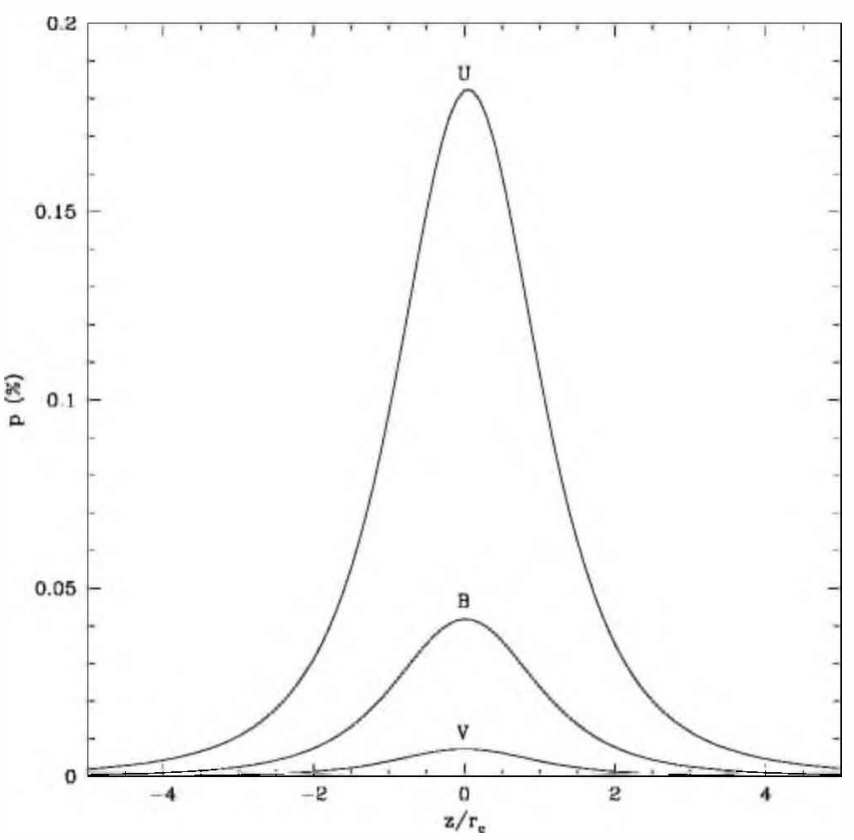

FIG. $11 h$

FIG. 11.-(a) Star (UIT 7) to nucleus (33" diaphragm) flux ratio in the UBV'bands and (b) resulting polarization due to an spherical dust cloud at the cluster nucleus with $\tau_{U}=0.5$, as a function of the coordinate along the line of sight (in core radius units) and assuming Rayleigh scattering.

and suggests the existence of excess polarization associated with the dark patch. We note that the geometric shape of the patch is not matched by that of the circular diaphragm. For this reason, a relatively large amount of light coming from its peripheral regions may produce a dilution effect on the intrinsic polarization eventually arising in the dark patch itself.

\section{DISCUSSION}

The analysis of the visual polarization of both the nonvariable giant stars and of the bright-star-free regions within $4^{\prime}$ of the cluster center shows a variation that can be attributed to the foreground dust along the line of sight to 47 Tuc. This variation is consistent with the polarizations observed on a larger angular scale, that is, stars between $4^{\prime}$ and $20^{\prime}$ from the center. An inspection of the $E_{B-V} I R A S$ calibrated map (Schlegel et al. 1998) over the same region, however, does not exhibit a systematic change of reddening, indicating that the observed polarization trend is more likely produced by a variation of the foreground polarization efficiency than by variable optical depth of the foreground interstellar dust.

After a vectorial removal of the foreground polarization, most of the residual values corresponding to the nonvariable stars are small, indicating that they are probably reflecting just the observational errors. However, there remains a detectable trend in the sense that the larger residuals are seen toward the south of the cluster, a direction opposite to that of the cluster proper motion. Krockenberger \& Grindlay (1995) have suggested that the X-ray structure observed around 47 Tuc could originate from the cluster motion and the interaction of its gas with the hot Galactic halo. The same mechanism, that is, ram pressure on dust grains, may lead to the formation of a tail-like structure, or a preferential direction along which dust grains leave the cluster (Pellizza González, Forte, \& Carpintero 2002). The spatial velocity components of 47 Tuc given in Table 3 of Krockenberger \& Grindlay (1995), based on absolute proper motions derived by Tucholke (1992) and Cudworth \& Hanson (1993), indicate that such a tail would be located toward the south of the cluster and be receding from the sky plane with an angle of about $55^{\circ}$. The lack of a regular pattern in the residual polarizations observed for some stars located toward the south of the cluster center might be explained as a consequence of optically thin dust locally illuminated by the stars themselves, or combined with backscattering of the cluster radiation as a whole. Backscattering properties for Galactic cirrus have been discussed, for example, by Jakobsen, de Vries, \& Paresce (1987). This possibility is also worth further analysis given the existence of a diffuse far-ultraviolet background, detected in Ultraviolet Imaging Telescope observations by O'Connell et al. (1997), that has a spatial scale length significantly different from that of the 47 Tuc stars. The dust scattering explanation was considered by these last authors and, in principle, ruled out because of the large dust mass required if "canonical " dust grains were present and mixed with the stars.

Another argument that may also support the existence of a tail-like structure comes from the appearance of the elongated structure detected on IRAS $12 \mu \mathrm{m}$ of the central regions of 47 Tuc by Gillett et al. (1988; see their Fig. 1b). This detection would imply relatively large dust equilibrium temperatures for an extended source. However, other radiation mechanisms, as, for example, very small particles transiently heated by absorption of single photons (Sellgren, Werner, \& Dinerstein 1983), may provide an explanation consistent with the polarimetric results discussed in $\S 8$, which also favor the presence of small particles.

There are other elongated or cirrus-like structures in the IRAS image. As mentioned above, one of these features appears to be connected to the red giant L1421. Gillett et al. 
(1988) point out that there is no compelling evidence for the connection of this high-temperature structure $(T=40 \mathrm{~K})$ with the cluster. However, the detection of intrinsic polarization in L1421 (see $§ 4$ ), a likely cluster member, suggests that this star is illuminating the elongated cloud. Furthermore, the spatial orientation of this tail-like structure is well aligned with the direction of the spatial proper motion of the cluster, corrected for the peculiar motion of the Sun and Galactic rotation, based on the proper-motion study by Cudworth \& Hanson (1993; see Table 3 of Krockenberger \& Grindlay 1995).

In contrast to the bright nonvariable red giants, almost all the observed variable stars show significant polarization excesses, a result consistent with those obtained by other authors and in the same direction as arguments already given by Frogel \& Elias (1988), in the sense that dust formation and mass loss driven by stellar pulsations are connected. In any case, and according to the very low mass-loss rates obtained by Ramdani \& Jorissen (2001), who also point out the lack of dust-enshrouded stars in 47 Tuc, the variable stars do not appear to be an important source of dust injection (and replenishment) into the cluster environment.

In turn, the multicolor polarization observations of the cluster nucleus do not show a detectable polarization maximum within the $U B V R I$ wavelength range, instead displaying a smooth increase toward the $U$ band. This trend cannot be matched with a Serkowski law, even if we make rather extreme assumptions about $\lambda_{\max }$ and for the $K$-parameters that would imply values of the total-to-selective extinction ratio as low as $R=2.0$ or less. Besides, the fact that the $U$ polarization exhibits significant changes with diaphragm aperture, and also a different polarization angle, rather suggests that the observed behavior could be a combination of the foreground interstellar polarization and another polarizing source that becomes more evident toward short wavelengths. The blue post-AGB star known as BS or UIT 7 may provide an adequate explanation, if this object is in fact illuminating some dust located in the cluster nucleus. Other possible origins, such as intrinsic polarization associated with the atmospheres of giant stars within the 47 Tuc nuclear region, can be ruled out, as their ultraviolet magnitudes, around $U=16-16.5$, are some 8 mag fainter than the integrated brightness of the nucleus within the $33^{\prime \prime}$ diaphragm.

The detection of dust within the nuclear region of another metal-rich globular cluster, NGC 6356, has been reported by Hopwood et al. (1998) on the basis of observations carried out with $850 \mu \mathrm{m}$ arrays. However, ISO observations of 47 Tuc itself (Hopwood et al. 1999) set a very low upper limit for the dust mass (assuming silicate or amorphous car- bon grains). In fact, the peak emission at $120 \mathrm{\mu m}$ is offset from the cluster center by $\sim 165^{\prime \prime}$ and a position angle of $118^{\circ}$ (see Fig. 2 in that paper). This position angle is bracketed by those corresponding to the bright-star-free regions Z12 and Z13 (although they are closer to the cluster center). An analysis of the integrated brightness of these regions, listed in Table 4, as a function of position angle shows that there is an overall decrease of the cluster surface brightness in that sector (including Z14 and Z1), which, on the average, is $0.5 \mathrm{mag}$ fainter than the mean defined by all the regions. This obscuration may arise in the same dust that reaches a flux of $\sim 35 \mathrm{MJy} \mathrm{sr}^{-1}$ in the ISO map. That this is probably a small angular scale structure is suggested by the lack of a similar feature in the lower resolution IRAS maps.

The origin of the disagreement between the $I S O$ results, which suggest that the nucleus is practically free of dust, and those coming from the polarimetric results presented in this paper remains unclear. A preliminary hypothesis assumes that the particles that produce polarization (in the cluster nucleus) and those that may give rise to the $120 \mu \mathrm{m}$ emission (at the offset position) have markedly different physical properties. The environmental conditions of these particles may indeed be very different in terms, for example, of the ionized gas density (Freire et al. 2001) and its effect on the grain survability.

High-precision color-magnitude diagrams (e.g., based on multicolor HST images) of individual stars may provide a good test for the presence of dust, although up to now the published photometric results are still somewhat noisy to reach definite conclusions. A dust cloud optical depth $\tau_{U}=0.5$ would imply a maximum blue extinction $A_{B}$ ranging from 0.4 to $0.2 \mathrm{mag}$ and a color excess $E_{U-V}$ between 0.18 and 0.41 (for a $\lambda^{-1}$ and a $\lambda^{-4}$ wavelength extinction dependence, respectively). These values are compatible with the red envelope of the $B$ versus $U-V$ color-magnitude diagram presented by Howell et al. (2000) for stars within $21 " .54$ of the cluster center.

A multicolor photometric program is currently under way (Faifer \& Forte 2002), focused on a number of dark patches that might be identified as knotty dust clouds with sizes smaller than 0.1 pe inside a more diffuse dusty environment in the 47 Tuc nuclear region.

The authors acknowledge the work of the CASLEO staff in developing the polarimeter and also their enthusiastic help during the several observing runs invested in this paper. Useful remarks by an anonymous referee are appreciated. This work was partially funded by CONICET and the Agencia Nacional de Promoción Científica y Técnica de la República Argentina.
Aurière, M., \& Leroy, J.-L. 1990, A\&A, 234, 164

Chun. M. S. \& Freeman. K. C. 1978, AJ, 83, 376

Clarke, D. \& Stewart, B. G. 1986. Vistas Astron., 29, 27

Cramer. N. 1984, A\&A. 132, 283

Cudworth. K. M., \& Hanson, R. B. 1993, AJ, 105. 168

Dixon. W. V. D.. Davidsen. A. F. \& Ferguson. H. C. 1995. ApJ. 454. L47

Draine, B. T., \& Lee, H. M. 1984, ApJ, 285, 89 (erratum 318, 485 [1987])

Faifer, F.. \& Forte. J. C. 2002. in preparation

Flower, P. J. 1996, ApJ, 469,355

Forte. J. C.. \& Méndez. M. 1988. AJ, 95, 500 1989. ApJ, 345, 222

Freire. P. C. Kramer. M.. Lyne. A. G.. Camilo. F., Manchester. R. N.. \&

D'Amico, N. D. 2001. ApJ, 557, L105

Frogel. J. A.. \& Elias. J. H. 1988, ApJ. 324, 823

\section{REFERENCES}

Gillett, F. C., de Jong, T., Neugebauer, G., Rice, W. L., \& Emerson, J. P. 1988. AJ 96, 116

Guhathakurta, P., Yanny, B.. Schneider, D. P., \& Bahcall, J. N. 1992, AJ 104, 1790 (erratum 105, 1607 [1993])

Hesser, J. E.. Harris, W. E., VandenBerg, D. A.. Allwright, J. W. B., Shott. P., \& Stetson, P. B. 1987, PASP, 99, 739

Hopwood, M. E. L., Evans, A., Penny, A.. \& Eyres, S. P. S. 1998, MNRAS. 301. L30

Hopwood, M. E. L., Eyres, S. P. S., Evans, A., Penny, A.. \& Odenkirchen. M. 1999. A\&A. 350. 49

Howell, J. H., Guhathakurta, P.. \& Gilliland, R. L. 2000, PASP, 112, 1200

Jakobsen. P.. de Vries. J. S.. \& Paresce, F. 1987. A\&A. 183. 335

Jura, M. 1978, ApJ, 223, 421

Kanagy, S. P.. \& Wyatt. S. P. 1978. AJ. 83.779 
Knapp, G. R.. Gunn, J. E.. \& Connolly, A. J. 1995, ApJ, 448, 195

Krockenberger, M. \& Grindlay, J. E. 1995. ApJ 4 451.200

Lee, S.-W. 1977, A\&AS, 27, 381

Lloyd Evans. T. 1974. MNRAS. 167.393

Magalhảes, A. M. Benedetti. E. \& Roland. E. H. 1984. PASP, 96, 383

Martin. P. G.. \& Shawl. S. J. 1981. ApJ. 251. 108

Martínez, E., Aballay, J. L., Marún, A., \& Ruartes, H. 1990, Bol. Asoc. Argentina Astron.. No. 36,342

Mathewson. D. S. \& Ford, V. L. 1970. MmRAS. 74, 139

Mayor. M.. et al. 1983. A\&AS. 54. 495

1984. A\&A. 134, 118

Minniti. D. Coyne. G. V.. \& Clariá. J. J. 1992. AJ. 103. 871

Minniti. D. Coyne, G. V. \& Tapia. S. 1990, A\&A. 236, 371

O'Connell. R. W.. et al. 1997. AJ. 114. 1982

Origlia, L., Scaltriti, F., Anderlucci, E., Ferraro, F. R., \& Fusi Pecci, F 1997. MNRAS. 292.753

Pellizza, L., Forte, J. C.. \& Carpintero, D. 2002, in preparation

Ramdani, A., \& Jorissen, A. 2001, A\&A. 372, 85
Reed, B. C. Hesser, J. E., \& Shawl, S. J. 1988, PASP, 100, 545

Roberts. M. S. 1960. AJ, 65, 457

Sawyer Hogg. H. 1973. Publ. David Dunlap Obs. 3 . No. 6

Schlegel. D. J.. Finkbeiner. D. P.. \& Davis. M. 1998. ApJ. 500.525

Sellgren, K. Werner. M. W. \& Dinerstein. H. L. 1983. ApJ 271. L13

Serkowski. K. 1973. in IAU Symp. 52. Interstellar Dust and Related Topics, ed. J. M. Greenberg. \& H. C. van de Hulst (Dordrecht: Reidel). 145

Serkowski, K., Mathewson, D. S. \& Ford, V. L. 1975. ApJ, 196. 261

Tucholke. H.-J. 1992. A\&AS. 93, 293

van de Hulst, H. C. 1957, Light Scattering by Small Particles (New York: Wiley)

Whittet, D. C. B., Martin. P. G., Hough. J. H., Rouse, M. F., Bailey, J. A. \& Axon. D. J. 1992. ApJ. 386. 562

Wilking, B. A., Lebofsky, M. J., \& Rieke, G. H. 1982, AJ, 87, 695

Witt. A. N.. Thronson. H. A.. Jr.. \& Capuano. J. M., Jr. 1992. ApJ. 393. 611 